\title{
$\rightarrow, \cdots$
}

$f+20$

$+4 \alpha^{2}+$

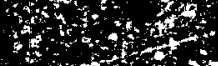

2

$+\tan +4,2$

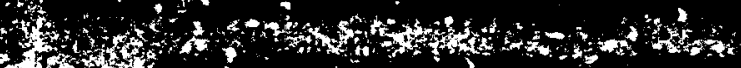

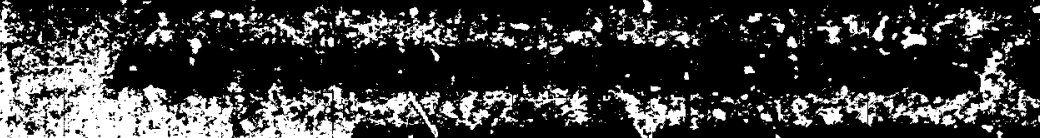

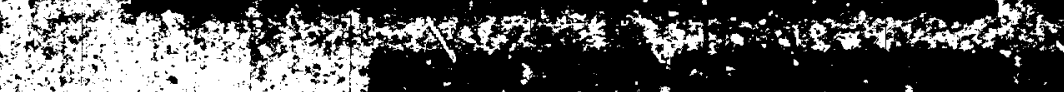

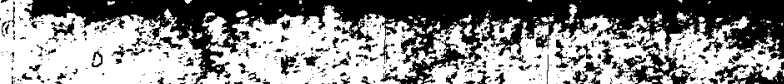

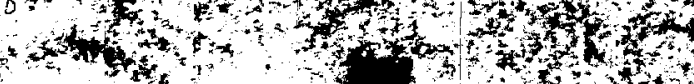

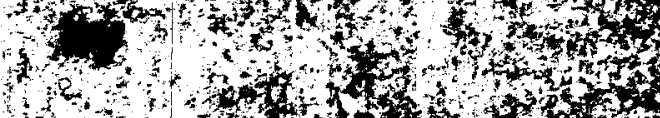

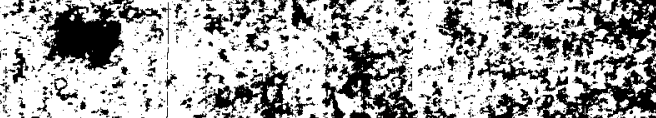

e.

tente

(3)

(f) $\div+\infty$

$34 t^{2}+2$

$4 x^{2}+1$

$$
1 \quad 2^{2}
$$

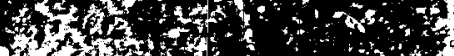

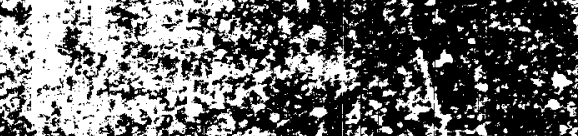

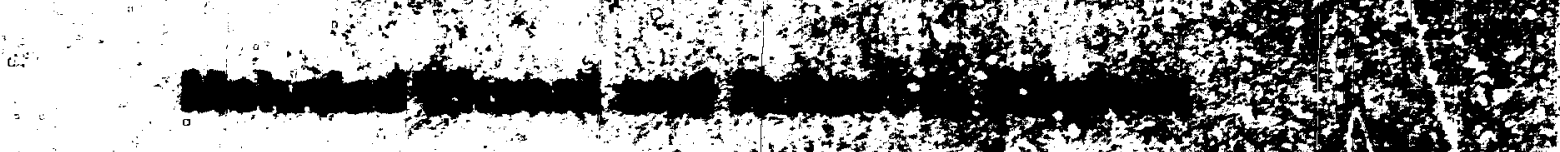
Why

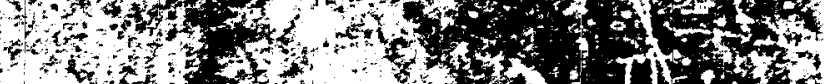

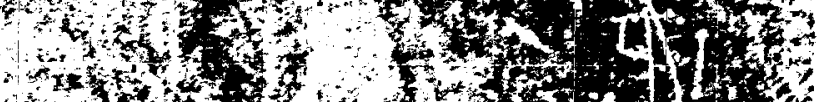

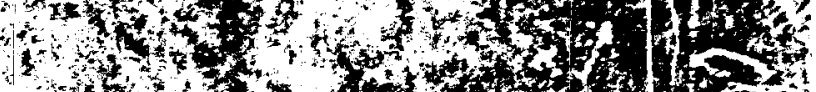

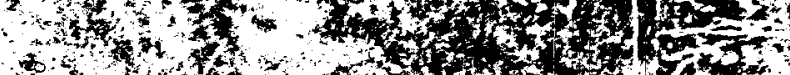

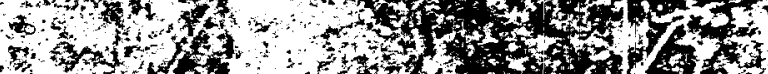

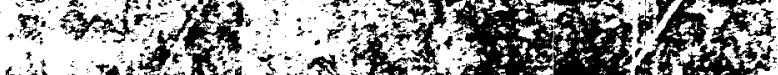

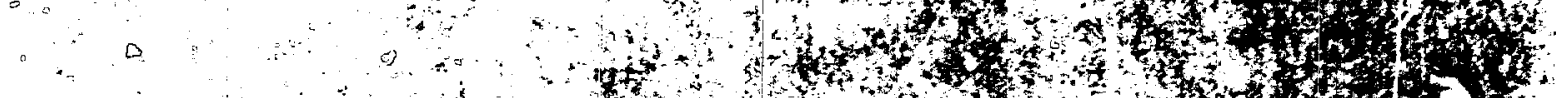
y)

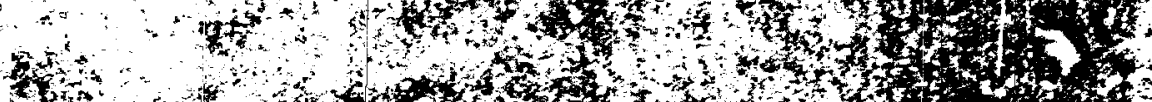

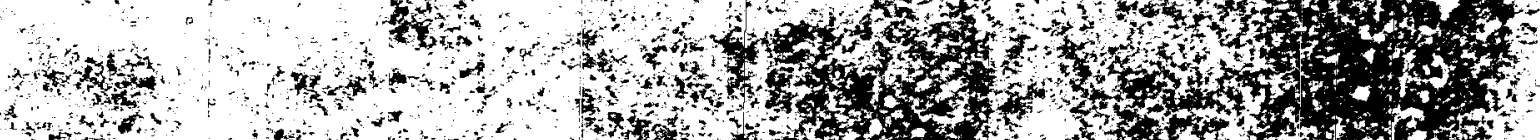

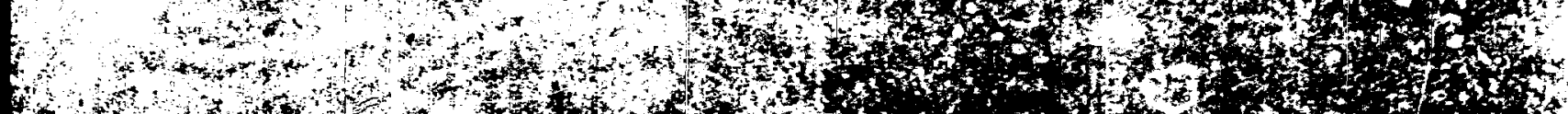
Hy (x) H.

tos s:

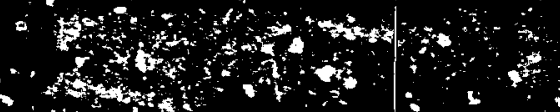
s. 
ANLIFPPITM-182

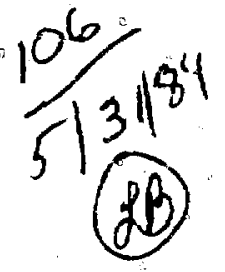

(1)

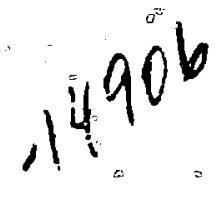

ANLIFPPITM-182

$D R * 0102-3$

\section{A NEW ONE.PHASE DUAL CONVERTER}

FOR SUPERCONDUCTING INDUCTIVE ENERGY STORAGE AND TRANSFER APPLICATIONS: THE ONE-PHÁSE INDUCTOR-CONVERTER BRIDGE

by

Mehrdad Ehsani and Robert L. Kustom

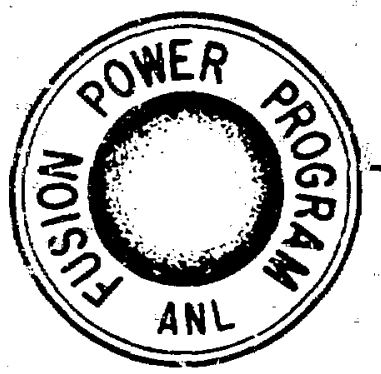

\section{FUSION POWER PROGRAM}

Argonne National Labohatory

9700 South Cass Avenus

Argonne, Ilinais 60439
Operated by

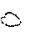

The University of Chicage for ine U.S. Departmont of Enorgy under Contrect W-31-109-Eng-38 
PORTIONS OF THIS REPORT ARE HLEGIBLE. it

has been reproduced from the best aratiable copy to oermit the b: eadest gossible availsbility
Distribution Category: Magnetic Fusion Energy (UC-20)

\author{
ANL/FPP/IL-132
}

DES4 012118

ARGONNE NATIONAL LABORATORY

9700 South Cass Avenue

Argonne, Illinois 60439

A NEW ONE-PHASE DUAL CONVERTER

FOR SUPERCONDUCTING INDUCTIVE ENEKGY STORAGE AND TRANSFER APPLICATIONS: THE ONE-PHASE IANUCTOR-CONVERTER BRIDGE

by

Mehrdad Ehsani* and Robert L. Kustom

Fusion Power Program

March 1984

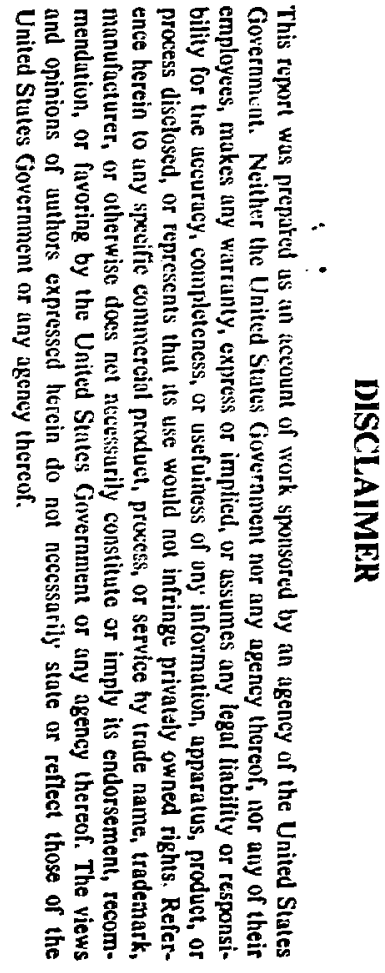

*Department of Electrical Engineering, Texas A\&M University, College, Station Texas 77843 
TABLE OF CONTENTS

$\underline{\text { Page }}$

ABSTRACT $\ldots \ldots \ldots \ldots \ldots \ldots \ldots \ldots \ldots \ldots \ldots \ldots \ldots \ldots \ldots \ldots \ldots \ldots \ldots \ldots \ldots \ldots$ vii

1. INTROUUCTION...................................... 1

2. THE ONE-PHASE INDUCTOR-CONVERTER BRIDGE............... 4

$2.1 \quad$ Circuit Operation........................... 4

$2.2 \quad$ Circuit Dynamic Analysis..................... I0

2.3 System Control............................ $14^{*}$

2.4 Methods of Phase and Frequency Shifting........... 17

2.5 Safe 0perating Lifts...................... 25

3. EXPERIMENTAL RESULTS........................... 29

3.1 Constant Phase Operation..................... 33

4. DISCUSSION AND CONCLUSIONS........................ 39

5. ACKNOWLEDGEMENT ............................... 43

6. REFERENCES ................................... 44

APPENDICES

1

2

3
Calculation of Average Coil Power............... 45

Calculation of the First Transient Step in

Frequency Shifting...........................

Operating Phase Threshold Calculation. 


\section{LIST OF FIGURES}

Page

1 Two basic power supply arrangements for inductive loads. (a) Power conditioner supplies the variable power directly fron the source. (b) Variable power is supplied by exchanging energy between the storage and load coils. A small supply compensates the system for the losses................ 2

3 One-phase ICB waveforms during the first few cycles with operating phase $\phi=90^{\circ} \ldots \ldots \ldots \ldots \ldots \ldots \ldots \ldots \ldots \ldots \ldots \ldots \ldots 7$

5 One-phase ICB waveforms at $i_{S}=0, i_{L}=i_{L m a x}$ and $\phi=90^{\circ}$

6 Idealized instantaneous: circuit model of the one-phase ICB......................................

7 Equivalent instantaneous circuit model of the one-phase ICB...................................

Instantaneous power vs control angle in one-phase ICB........

9 Simple bang-bang control algorithm....................

10 Three-step transient sequence phase shifting of

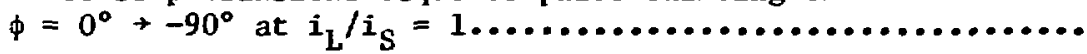

11 Three-step transient sequence phase shifting of $\phi=90^{\circ} \rightarrow-90^{\circ}$ at $i_{L} / i_{S} \cong 0$

12 Two-step transient sequence frequency shifting;

frequency increase..................................

13 Two-step transient sequence frequency shifting;

frequency decrease................................

14 Two-step transient sequence frequency shifting. Transient sequence began on the lagging converter...................

15 Double four-step composite phase-frequency shifting to maintain constant phase...............................

16 Example of incorrect capacitor commutation voltage due to excessive phase difference.............................

17 Plot of acceptable operating phase thresholds in one-phase ICB. 
18 Circuit diagram for the three-phase ICB................. 30

19 Example of $180^{\circ}$ phase shift in two $90^{\circ}$ segments

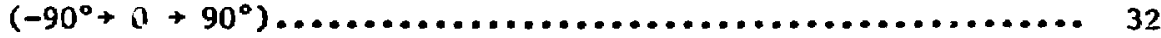

20 Coil current waveforms through a fuli energy transfer at

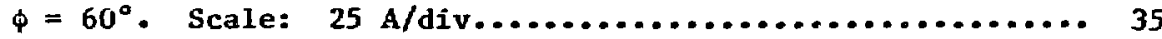

21 Capacitor voltage during the first cycles of operation. Scale: $50 \mathrm{v} /$ div................................... 35

22 Capacitor and inductor voltage waveforms during $t \cong 1 \mathrm{~s}$ at $\phi=60^{\circ}$. Top: $v_{S}$, Middle: $v_{C}$, Bottom: $v_{L} \ldots \ldots \ldots \ldots \ldots . . .35$

23 Capacitor and inductor voltage waveforms during $t \cong 2.5 \mathrm{~s}$ at $\phi=60^{\circ}$. Top: $v_{S}$, Middle: $v_{0}$ = Bottom: $v_{L} \ldots \ldots \ldots \ldots \ldots 36$

24 Capacitor and inductor voltage waveforms during $t \cong 4 \mathrm{~s}$ at $\phi=60^{\circ}$. Top: $v_{S}$, Middle: $v_{C}$, Bottom: $v_{L} \ldots \ldots \ldots \ldots \ldots . . .36$

25 Capacitor and inductor voltage waveforms during $t \cong 2 \mathrm{~s}$ at $\phi=90^{\circ}$. Top: $v_{S}$, Middle: $v_{C}$, Bottom: $v_{L} \ldots \ldots \ldots \ldots \ldots . . . .38$

26 Capacitor voltage during a $\phi=90^{\circ}$ to $-90^{\circ}$ phase shift..... 38

27 Coil voltages during a $\phi=90^{\circ}$ to $-90^{\circ}$ phase shift........ 38

28 Load coil voltage startup transient while the load parallel resistor is on.............................. 40

29 Inductor voltages at the beginning of operation showing the first phase shift transient on the load voltage.

Top: storage voltage, Bottom: load voltage................

30 An exaggeraced example of capacitor voltage self balancing in the one-phase ICB. (Long SCR gate pulses starting from the $i_{g}$ impulse instants are assumed.) .................. 42

A-1-1 Plot of $S q(t)$ and $\operatorname{Tr}(t)$ at an Arbitrary Phase Shift....... 47

A-3-1 Capacitor component voltages in the vicinity of $\mathrm{S}_{22} \mathrm{~S}_{23}$

A-3-2 Capacitor component voltages in the vicinity of $\mathrm{s}_{21} \mathrm{~s}_{24}$ commutation instant.................................. 


\title{
A NEW ONE-PHASE DUAL CONVERTER FOR SUPERCONDUCTING INDUCTIYE ENERGY STORAGE AND TRANSFER APPLICATIONS: THE ONE-PHASE INDUCTOR-CONVERTER BRIDGE*
}

by

Mehrdad Ehsiani and Robert L. Kustom

\begin{abstract}
This report presents the results of theoretical and experimental development of the one-phase Inductor-Converter Bridge (ICB). The basic operating principles of the circuit are described followed by a theoretical treatment of the dynamics and control of the system. The successful results of the first experimental operation ind control of the one-phase ICB are presented and explained. Finally, a discussion of some of the interesting transient and steady state behavior of the circuit, along with some of its unique features, is given.
\end{abstract}

* Fork supported by the U. S. Department of Energy. 


\section{INTRODUCTION}

Superconducting magnet coils are being increasingly used for energy storage and generation of high magnetic fields iri research. Superconducting magnets are not oniy capable of producing very high fields, but are also very energy efficient due to their low losses. Some prominent examples of large superconducting coils contemplated or under design are the equilibrium field (EF) coils of Argonae National Laboratory Tokamak Experimental Power Reactor ${ }^{(1)}$ and Wisconsin Superconductive Energy Storáge coil. (2) Examples of medium size superconducling cojls in use today include the accelerator beam control coils at Fermi National Laboratory ${ }^{(3)}$, the experimental power system stabilizer coil of Los Alamos Nationai Laboratory ${ }^{(4)}$, and many others.

From the electrical terminals, the superconducting coil is a high $Q$ inductor, capable of storing large amounts of energy. To supply this energy efficiently, the power supply should also have low losses and be capable of reversible power control. The need for efficient reversible power control is particularly pressing in repetitively energized magnets. Solid state switching power supplies have been utilized in recent years $(5,6,7)$ for this purpose. The low conduction losses and fast switching of solid state power switches, together with their increasingly higher power ratings, $*$ make these supplies favorable for superconducting magnet applications onto the future. Where repetitive two-quadrant (bidirectional) power control is required, power supply circuits utilizing another superconducting energy storage coil $(7,8)$ as a buffer have been suggested (Fig. 1). The main advantage of these circuit arrangements is that high power oscillations, required by the load, are supplied by the energy storage coil. Thus; a relatively small power generator or utility link can be used for the initial energization of the storage coil and steady state system loss compiensation. Two prominent examples of circuits utilizing an energy storage buffer are the Flying Capacitor (FC) arrangement suggested by Dustman ${ }^{(6)}$ and the Inductor-Converter Bridge (ICB) suggested by

*Silicon Controlled Rectifiers (SCR) of 10 WNA rating and higher can presently be obtained. Through the gate turn off devices, such as gate turn off (GTO) SCR's and power transistors, are also growing in ratings, although not quite up to the SCR levels. Series and paraliel connection of solid state switches produces composite switches of much higher ratings. 

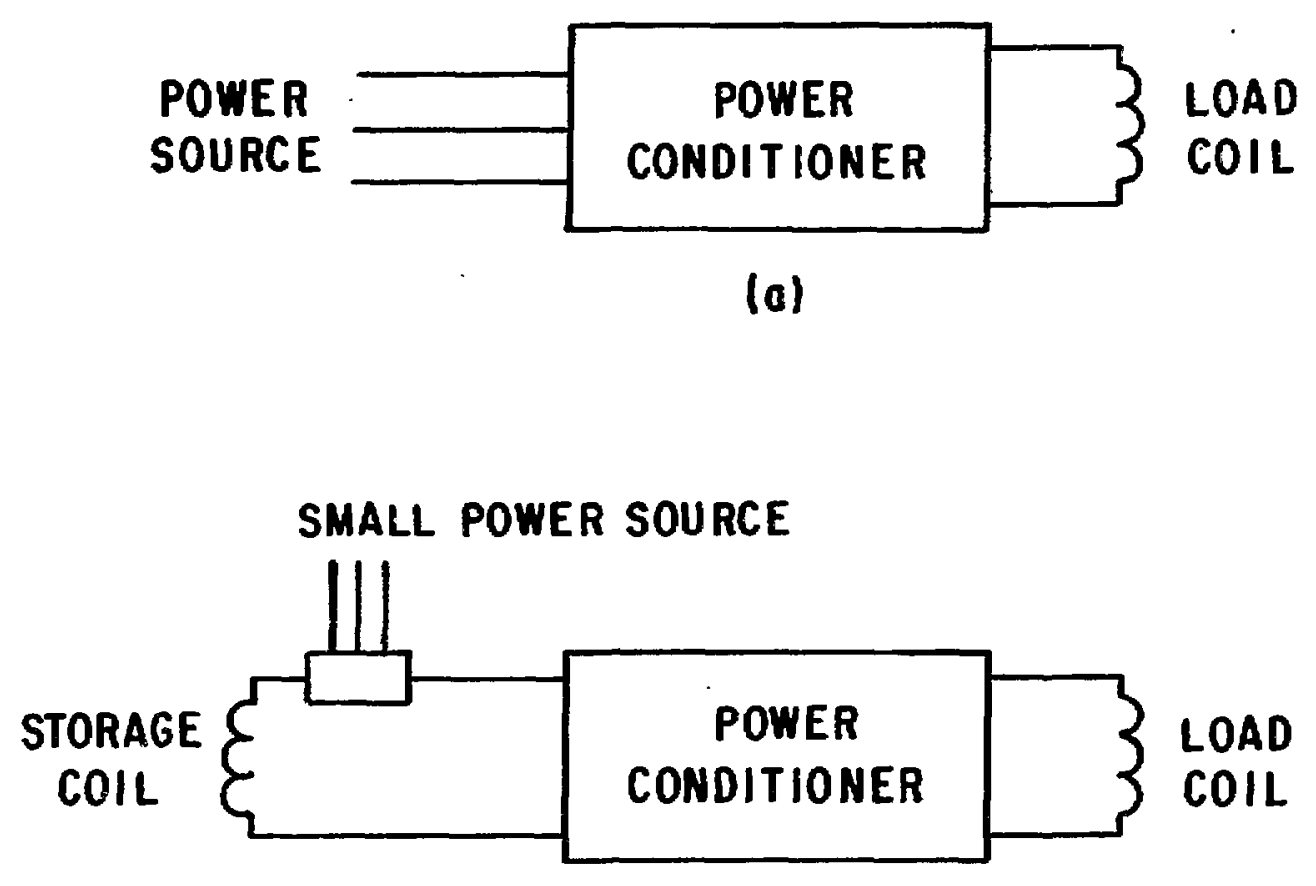

(b)

Fig. 1. Two basic power supply arrangements for inductive loads.

(a) Power conditioner supplies the variable power directly from the source.

(b) Variable power is supplied by exchanging energy between the storage and load coils. A small supply compensates the system for the losses. 
Peterson and Mohan(5). An extensive study of these circuits has been presented in a book by Kustom. (7)

This writing presents the results of theoretical and experimental work on a new one-phase dual converter for magnet supply which is in the class of ICB circuits. The circuit was first suggested by Ehsani and Kustom. (9) Recent experimental verification of the circuit was done by the authors under the auspices of Argonne National Laboratory and Wisconsin Applied Superconductivity Laboratory at University of Wisconsin, Madison. 


\section{THE ONE-PHASE INDUCTOR-CONVERTER BRIDGE}

The dual converter described in this report is the one-phase version of the Inductor-Converter Bridge (ICB) which was originated by Peterson and his colleagues and was further developed by the present authors. The unique characteristics of the operation and control of one-phase ICB are described below.

\subsection{Circuit Operation}

Figure 2 shows the basic one-phase ICB circuit. The storage coil, $\mathrm{L}_{\mathrm{S}}$, and load coil, $L_{L}$, are each connected to a full wave one-phase converter as shown. The ac lines of the converters are connected in parallel with the capacitor $C$. The switching sequence on the storage converter is $\mathrm{s}_{11} \mathrm{~s}_{14}, \mathrm{~s}_{12}$ $\mathrm{s}_{13}, \mathrm{~s}_{11} \mathrm{~s}_{14}$, etc. The same switching sequence is used on the load converter. However, corresponding switching events on the storage and load converters need not be synchronized. Leading timing (leading phase) of the load converter relative to the storage converter will cause a net energy transfer from storage to load coil. Similarly, lagging load converter timing (lagging phase) will cause net energy removal from the load coil. The capacitor on the ac side of converters serves two functions. It temporarily stores the energy which is transferred from one coil to the other in each converter cycle. It also supplies the reverse voltages required for commutation of current between $s_{1} s_{4}$ and $s_{2} s_{3}$ switches on each converter. Although the above operation is generally similar to other ICB circuits, an instantaneous double short circuit occurs across each inductor during commutation twice a cycle which does not happen in the previously tested ICB systems. For example, $s_{11} S_{14}$ conduction is turned off by firing $s_{12} s_{13}$. During commutation, all $s_{11}, s_{12}, s_{13}$ and $s_{14}$ switches are in conduction and a double path short eircuit exists on $\mathrm{L}_{\mathrm{S}}$. A positive voltage on the capacitor $\mathrm{C}$ is required to commutate $s_{11}$ and $s_{14}$ off while maintaining conduction in $s_{12}$ and $s_{13}$. However, the above double short conduction pattern is not inherently unstable because short circuit across a large inductor maintains a constant current. Switch $s_{11}$ is turned off by the commutation loop consisting of $s_{11}$, $S_{12}$ and $C$. With a voltage positive on the dotted side of $C$, shown in Figure 2 , reverse $s_{11}$ current will flow in the mentioned loop. When this commutation current equals the forward current, $i_{S}$, carrled by $s_{11}$, this SCR will open as 


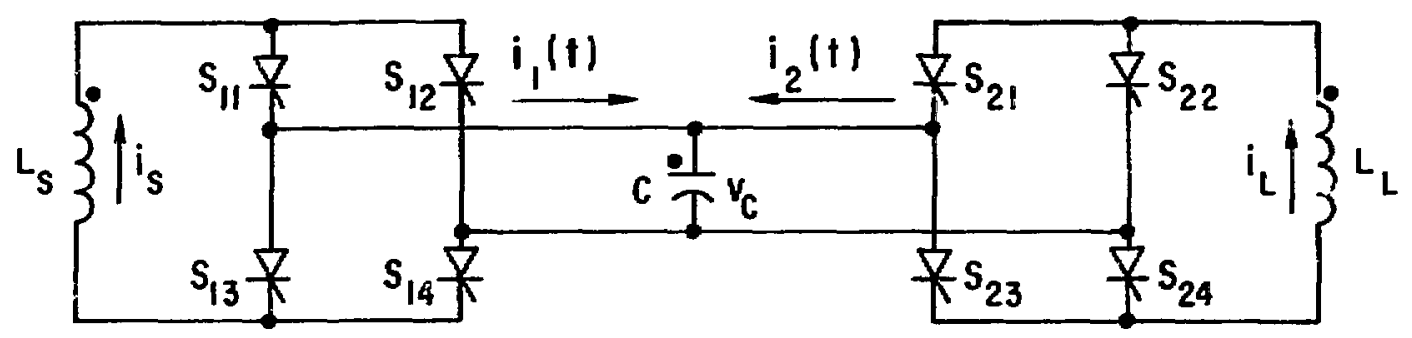

Fig. 2. Circuit diagram of the one-prase ICB. 
in a reverse biased diode. Note that the inductance and resistance of the above commutation loop is very smail by design. Therefore, small capacitor voltages can set up very high amplitude commutation currents which are sufficient for diverting $i_{S}$ away from $s_{11}$. A similar commutation current occurs in the loop consisting of $s_{14}, s_{13}$ and $c$ which turns off $s_{14}$. The duration of this commutation, called overlap, is very short relative to the converter cycle. It is noteworthy that significant electromagnetic noise is radiated from these high current short pulse loops which is common in SCR circuits. Four such double commutations take place in each cycle of the onephase $\mathrm{ICB}$. It is evident from the above discussion that correct capacitor voltage polarity must be present for successful commutation oí one pair of SCR's to the next in each converter. The limitations imposed by this voltage polarity will be considered later in this report. However, it is a simple matter to verify on the diagram of Fig. 2 that turning off $\operatorname{SCR}^{2} \mathrm{~s}^{*} \mathrm{~s}_{11} \mathrm{~s}_{14}$ and $\mathrm{s}_{21} \mathrm{~S}_{24}$ requires positive capacitor voltage, with the dotted side signifying positive voltage convention. Similarly, turning off SCR's $s_{12} s_{13}$ and $s_{22} s_{23}$ requires negative capacitor voltage. Therefore, an initial voltage; e.g., v $<0$ is placed on the capacitor and the converters are always started with the same SCR pairs; e.g., $\mathrm{s}_{11} \mathrm{~s}_{14}$ and $\mathrm{S}_{21} \mathrm{~S}_{24} \cdot$

The circuit usually begins operation with the storage inductor fully charged and the load inductor uncharged. The converter switching frequency is chosen such that the total storage energy is transferred to the load over several thousand converter cycles. Therefore, each converter cycle transfers a very small fraction of the system energy. Thus, the coil currents are almost unchanged during a single converter cycle. During the first few cycles of operation the capacitor current is almost entirely from the stcrage converter, $i_{1}(t)$ in Fig. 2. This current waveform along with the resulting capacitor voltage is shown in Fig. 3. The waveforms in this figure are for the case of load converter switching leading the storage converter by a quarter cycle or a phase difference of $\phi=90^{\circ}$. The impulse gate currents, $i_{g}$, show the timing of each SCR pair on the storage and load converters. Note that for converter operation starting with $S_{11} s_{14}$, a negative capacitor

\footnotetext{
${ }^{*} \phi>0$ convention signifies load converter leading the storage converter. Similarly, $\phi<0$ signifies load converter lagging.
} 


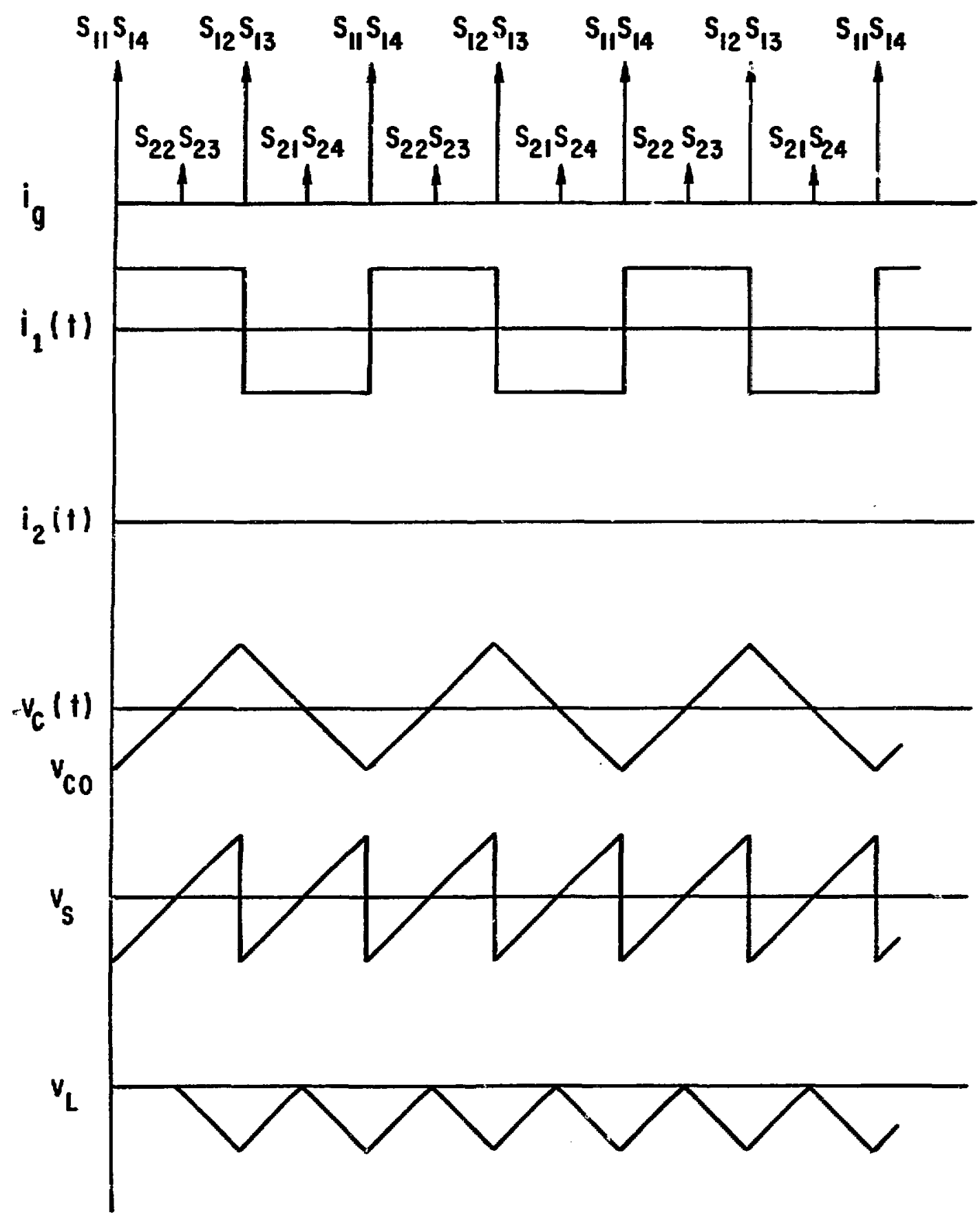

Fis. 3. One-phase ICB waveforms during the first few cycles with operating phase $\phi=90^{\circ}$. 
precharge voltage, $v_{c o}$ is present. Balanced capacitor voltage oscillation requires that $v_{C O}$ be precisely equal to peak negative capacitor voltage calculated below.

$$
v_{\text {Co }}=-\frac{T i_{\text {SO }}}{4 C}
$$

where $T$ is the converter period and $I_{S O}$ is the initial storage coil current. however, it will be shown that any capacitor voltage unbalance due to precharge voltage inaccuracy will decay to zero. In other words, the circuit can automatically correct for capacitor voltage errors. This notion may provide several benefits as will be discussed later. The storage and load coil voltages, $v_{S}$ and $v_{L}$, shown in Fig. 3 are with the positive voltage convention being the dotted side of the coils in $\mathrm{rig}$. 2. It is observed that the initial average voltages on the storage and load coils are zero and negative, resfectively. This will be shown more clearly in the theoretical analysis of the system. It is also interesting tc note that $s_{11} s_{14}$ and $s_{12} s_{13}$ commutations have ample capacitor voltages of the right polarity. However, $S_{2 i}$ $\mathrm{S}_{24}$ and $\mathrm{S}_{22} \mathrm{~S}_{23}$ have to commutate when the capacitor voltage is almost zero. Therefore, $i_{S}=i_{S O}$ and $i_{L}=0$ is a threshold state for successful commutation at $\phi=90^{\circ}$. It will be shown analytically that the commutation voltage will progressively improve as $i_{L}$ builds up.

Figure 4 shows the circuit waveforms when $i_{L}=i_{S}$. For a system with equal storage and load inductances $\left(L_{S}=L_{L}\right)$ this is the point of equal energy in the coils. The capacitor voltage component due to stcrage converter, $i_{l}$, is shown as $\mathrm{v}_{\mathrm{Cl}}$ while the component due to the load converter, $i_{2}$, is shown as $v_{C 2}$ - The actual capacitor voltage, $v_{C}$ is the sum of these two components. The coil voltages are derived from $v_{C}$ and by observing which sCR pairs are conducting on each converter at a given time. It is observed that a net positive voltage is present on $L_{S}$ and a negatije net voltage exists on $\mathfrak{L}_{L}$ • These voltages are responsible for extracting net erergy from $L_{S}$ and adding to $\mathrm{L}_{\mathrm{L}}$. Note, however, that oscillating coil voltages against dc coil currents produce oscillating instantaneous coll powers. Also note that ample capacitor commutation voltages of the correct polarity exist for $s_{1} s_{4}$ and $s_{2} s_{3}$ commutations on both converters. For completeness, the circuit waveforms at the end of energy transfer, where $i_{S}=0$ and $i_{L}=i_{L m a x}$, is shown in Fig. 5 . It is seen that capacitor voltage, $v_{C}$ is entirely determined by the lcsd 


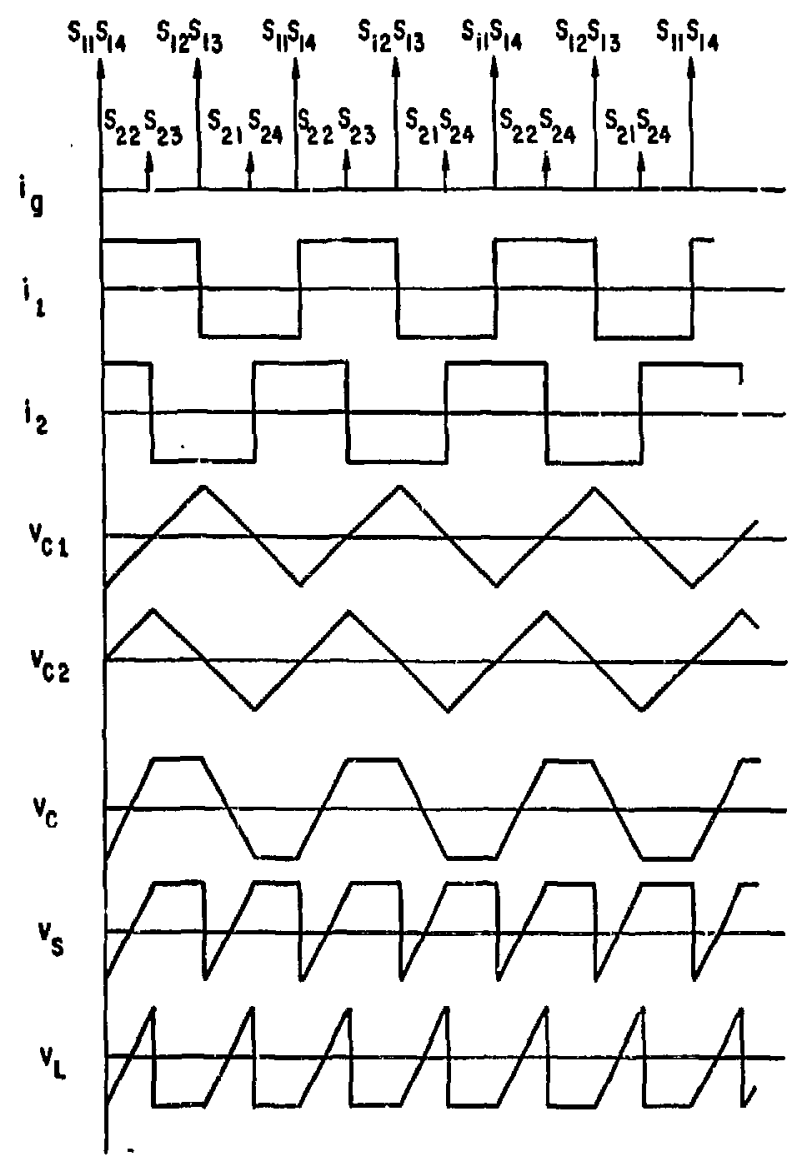

Fig. 4. One-phase ICB waveforms at $I_{L}=I_{S}$ and $\phi=90^{\circ}$.

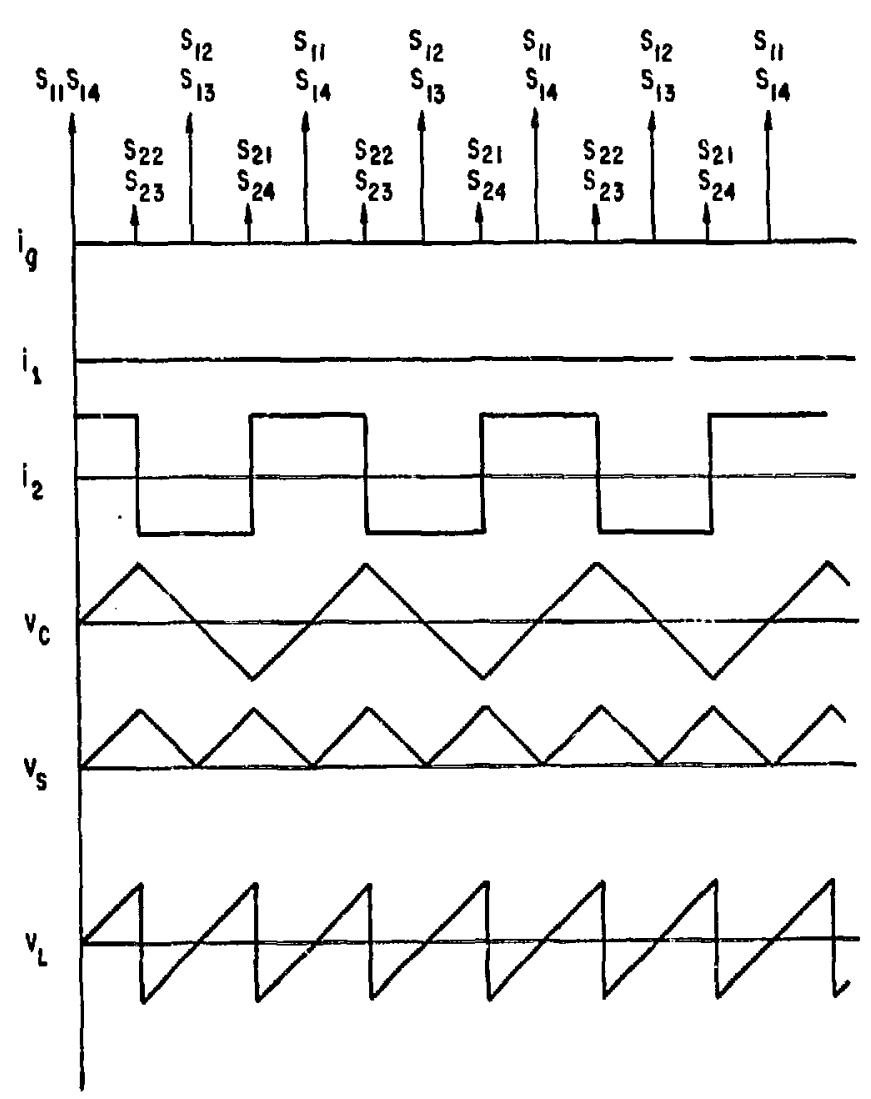

Fig. 5. One-phase ICB waveforms at $i_{S}=Q, I_{L}=I_{L \max }$ and $\phi=$ 
converter current, $I_{2}$. The coil voltages show a high net voltage on $L_{S}$ and a zero net voltage on $L_{L^{*}}$ Circuit waveforms at numerous other energy states, $i_{L} / i_{S}$ and phase angles, $\phi$, may similarly be drawn.

\subsection{Circuit Dynamic Analysis}

A detailed calculation of all multiphase ICB circuits, including the onephase, was presented in Reference 9. A summary of the analysis is included in this report. Figure 6 shows an idealized wodel of the one-phase ICB. In this model the converter SCR's have been replaced by their ideal switch function. Since the coil currents do not change significantly during one converter cycle, the coils have been replaced by ideal constant current sources over a short period of time. The current value of these sources is equal to the average coil currents during the converter cycle of interest. The converter switches of Fig. 6 simply invert their respective dc currents and produce square wave ac curreits $i_{1}(t)$ and $i_{2}(t)$. An equivalent.idealized model of the circuit may be drawn from the ac lines point of view as shown in Fig. 7. In this model the capacitor and its voltage is left unchanged. However, the dc current source and the converter switches have been replaced by the their resulting ac current source function. An example of $i_{1}(t)$ and $i_{2}(t)$ current waveforms for the case of $\left(i_{L} / i_{S}\right)=1$ and $\phi=90^{\circ}$ was shown in Fig. 4. In the circuit of Fig. $?$ it is simple to calculate average power from one current source to the other, over one converter cycle. The only complication is that $i_{1}$ and $i_{2}$ are periodic square waves. Therefore, the calculation is done on the Fourier components of these currents. The actual caículation is shcwn in Appendix 1 and the resulting average power over one converter cycle is repeated below.

$$
\left\langle p s^{\rangle}=\sum_{n=1}^{\infty} \frac{4 i s^{i} L}{n^{3} \pi^{2} \omega C}\left[1-(-1)^{n}\right] \sin r: \gamma\right.
$$

In Eq. $(2-1)\left\langle p_{S}\right\rangle$ symbolizes average storage coll output power over one converter cycle, $i_{S}$ and $i_{L}$ are the average coil currents over the same cycle, $i$ is the angular frequency of converter operation, and $\phi$ is the load converter advance angle.

A closed forn expression for $\left\langle\mathrm{p}_{S}\right\rangle$ was derived in Reference 9 in which a nes set of switching functions, named quadrometric functions, was used. A 


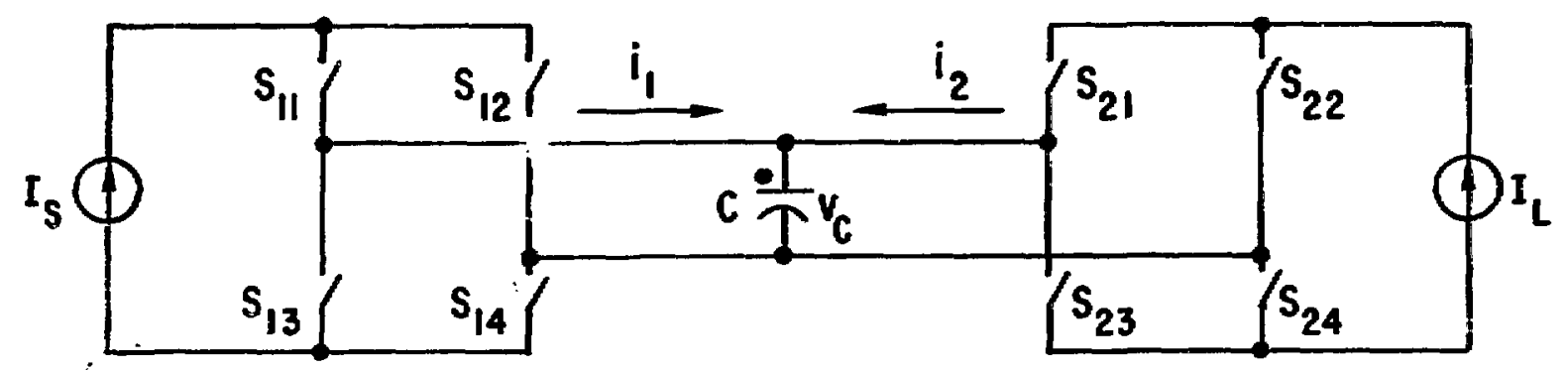

Fig. 6. Idealized Instantaneous circult model of the one-phase ICB.

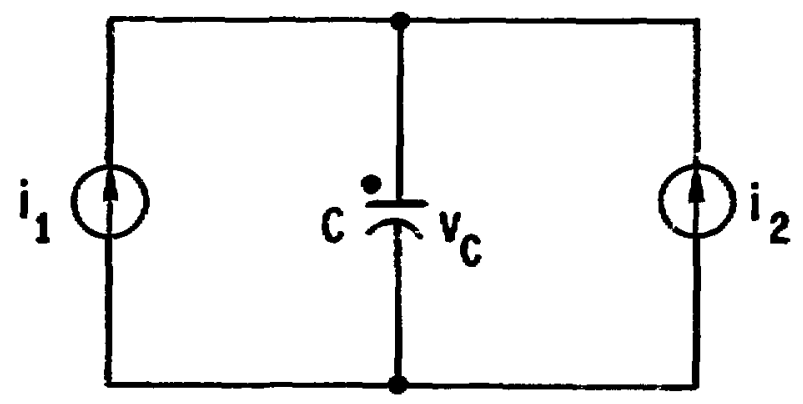

Fig. 7. Equivalent instantaneous circuit model of the one-phase ICB. 
Summary of this derivation is presented in Appendix $I$ and the result is repeated below.

$$
\left\langle p_{S}\right\rangle=\frac{{ }^{i} S^{i} L}{\omega C}\left(\phi-\phi^{2} / \pi\right)
$$

Since the ICB systems state $i_{L} / 1_{S}$ does not change appreciably over one cycle, the one cycle average power shown in $\mathrm{Eq}$. (2-1) a:td (2-2) may be approximately termed instantaneous ICB power as a function of phase.* A plot of this instantaneous power vs the operating phase angle is show in Fig. 8 .

The instantaneous power is also a function of tie instantaneous coil currents $i_{S}$ and $i_{L}$ as shown below

$$
\left\langle p_{s}\right\rangle=\frac{{ }^{i} s^{i} L}{\omega C}\left(\phi-\phi^{2} / \pi\right)
$$

Here again the instantaneous coil current is taken to be approximately the coil current anywhere during a given cycle. The iifferential equations for coil currents as a function of time are based on the instantaneous conservation of power ${ }^{(9)}$ :

$$
\begin{aligned}
& \text { storage output power }=\left\langle\mathrm{P}_{S}\right\rangle=-\frac{d}{d t}\left(\frac{l}{2} L_{S}{ }^{i}{ }^{2}\right) \\
& \text { load input power }=\left\langle p_{S}\right\rangle=\frac{d}{d t}\left(\frac{1}{2} L_{L_{L}}{ }^{2}\right) \\
& i_{S}(0)=I_{S O}=\text { initial storage current } \\
& \left.L_{L} \frac{d i_{L}}{d t}\right|_{t=0}=0=\text { initial load voltage over one sycle. }
\end{aligned}
$$

Substituting Eq. (2-3) in (2-4) and (2-5) and solving the differential equations with the initial conditions of Eq. (2-6) and (2-7) will result in the following instantaneous coil current expressions as a function of time.

$$
i_{S}(t)=i_{s o} \cos \frac{k}{\sqrt{L_{s} L_{L}}} t
$$




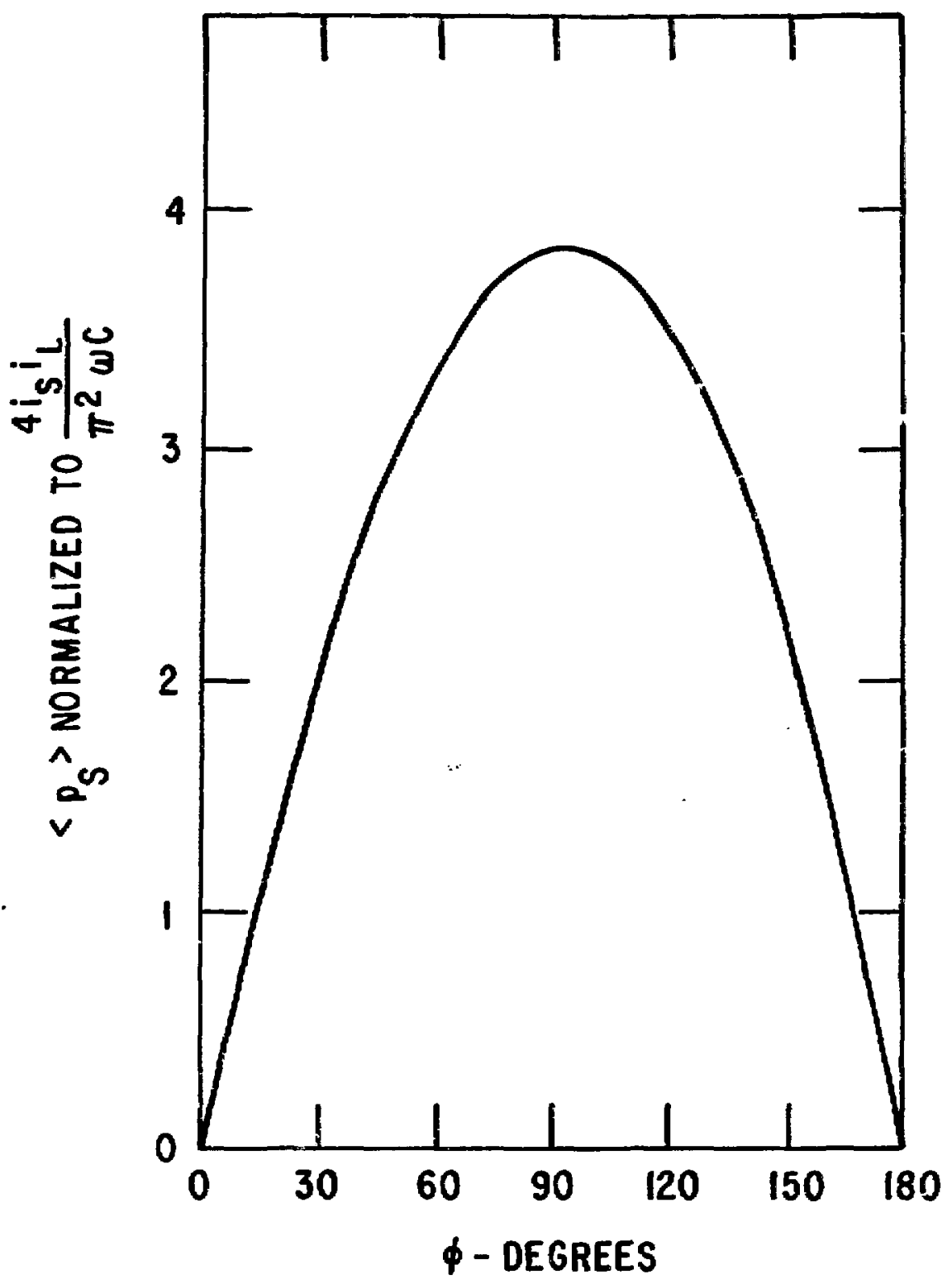

Fig. 8. Instantaneous power vs control angle in one-phase ICB. 
where

$$
i_{L}(t)=i_{\text {SO }} \sin \frac{k}{\sqrt{L_{S}^{L_{L}}}} t
$$

$$
i \triangleq\left(\phi-\phi^{2} / \pi\right) / \omega c
$$

The explicit time function of average power, from cycle to cycle, may be derived by substituting Eq. (2-8) and (2-9; In Eq. $(z-3)$. The result is

$$
\left\langle p_{S}\right\rangle(t)=\frac{1}{2} k i_{S O}^{2} \sqrt{L_{S} / L_{L}} \sin \frac{2 k}{\sqrt{L_{S}{ }^{i} L}} t
$$

The average coil voltages, from cycle to cycle, may be derived, respectively, by substituting $\mathrm{Eq} \cdot(2-8)$ and $(2-9)$ in

$$
\langle v\rangle(t)=L \frac{d}{d L} i
$$

The results are

$$
\begin{aligned}
& \left\langle v_{S}\right\rangle(t)=k i_{s o} \sqrt{L_{S} / L_{L}} \sin \frac{k}{\sqrt{L_{S} L_{L}}} t \\
& \left\langle v_{S}\right\rangle(t)=k i_{s o} \sqrt{L_{S} / L_{L}} \cos \frac{k}{\sqrt{L_{S} L_{L}}} t
\end{aligned}
$$

It is important to recognize that the actual instantaneous coil currents, $1(t)$, and the average coil currents from cycle to cycle, $\langle 1\rangle(t)$ are almost identical. Therefore, Eq. (2-8) and (2-9) approximate $i_{S}(t)$ and $i_{L}(t)$. However, the instantaneous coil voltages, $v(t)$ and power, $p(t)$ are quite different from the average values from cycle to cycle, $\langle v\rangle(t)\langle p\rangle(t)$, which were calculated above.

\subsection{System Control}

The one-phase ICB is not only intended for efficient repetitive charging and discharging a magnet coil but also for control of the load magnetic field based on a reference or command signal. Therefore, the control system would

typically be configured in closed loop. It has baen shown ${ }^{(9)}$ that a bang-bang 
control strategy, based on the coil current error signal is a time optimal, robust, and simple method of controlling the three-phase ICB. The same arguments hold for the one-phase ICB. A discrete time flow chart of the bangbang control algorithm is shown in Fig. 9. The algorithm acquires the present values of load current, $i_{L}\left(t_{K}\right)$, reference current, $i_{R}\left(t_{K}\right)$ and phase, $\phi\left(t_{K}\right)$. If the present load current is less than the present reference, a next value of phase command, $\phi\left(t_{\mathrm{K}+1}\right)$ for maximum input power to the load is issued." Conversely, a next value of phase for maximum output power from the load is issued if $i_{L}\left(t_{K}\right)<i_{R}\left(t_{K}\right)$. The phase angle is unchanged if no load current error is sensed, $i_{L}\left(t_{K}\right)=i_{R}\left(t_{K}\right)$. It is a simple matter to calculate the maximum power phase angle from Eq. (2-3). This value can also be seen from the plot of Fig. 8 to be $\phi= \pm 90^{\circ}$. Therefore, a one-phase ICB is bang-bang controlled at $\phi=90^{\circ}$ and $\phi=-90^{\circ}$. The fortuitous equality of control boundaries, $\pm 90^{\circ}$, in three-phase and one-phase ICB systems simplified the cxperimental modifications of our prototype ICB which is presented later.

Equation (2-3) shows that converter frequency, $w$, can also control the load power. However, since frequency is always positive. the sign of load power cannot be controlled by frequency. Furthermore, the range of frequency variation is greatly limited from the lower side by maximum system voltage ratings and from the upper side by the switching speed of the SCR's. In practice, the operating frequency is much further limited by the acceptable switching losses from the upper side and a variable lower limit. The variable lower limit depends on the $i_{L} / i_{S}$ state of the system. It can be easily shown that the largest peak capacitor voltage occurs when $\phi=0$ and $\left(i_{S}+i_{L}\right)$ is a maximum. This peak voltage can be calculated by an equation similar to Eq. $(2-0)$ :

$$
v_{C \max }=\frac{T{ }_{C \max }}{4 C},
$$

where

$$
i_{\text {max }}=\left(i_{s}+i_{L}\right) \text { max }
$$

*Maximum power commands will induce the highest rates of rise and fall of load cuirent. Therefore, this strategy responds to a step change of reference in the shortest time. 


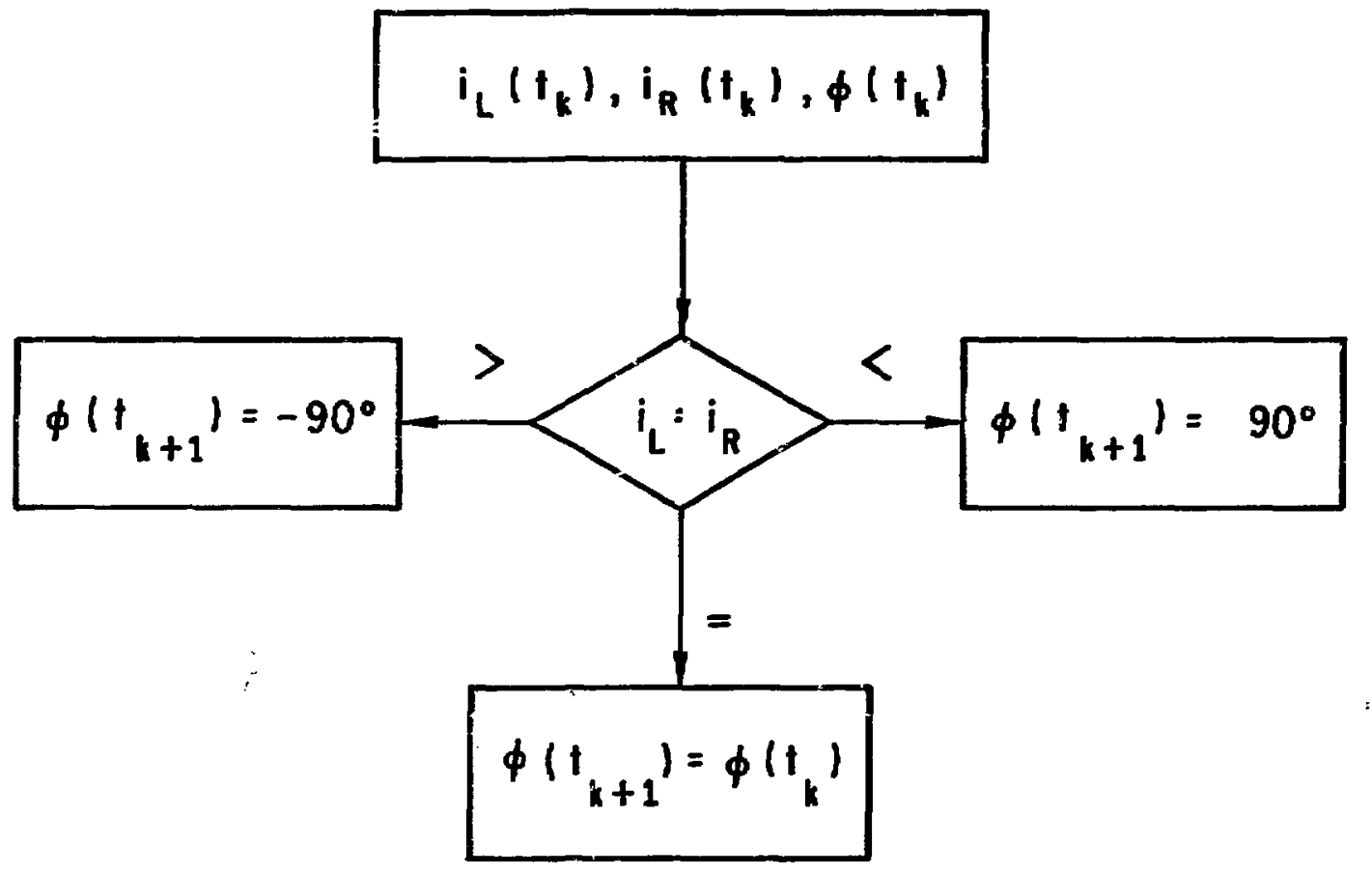

Fig. 9. Simple bang-bang control algorithm. 


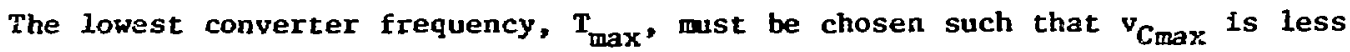
than system voltage rating. In this case the lower limit of the control frequency is independent of the $i_{L} / i_{S}$ state. However, this would be a conservative lower limit. In all other $i_{L} / i_{S}$ states, we have $\left(i_{S}+i_{L}\right)\left\langle\left(i_{S}+\right.\right.$ $i_{L}{ }{ }_{\max }$. Therefore, the ICB system can usually be operated at yet lower frequencies withour exceeding $v_{\text {Cmax }}$ rating. If such an extrene lower limit of frequency is chosen, it will have to be updated based on the present value of ${ }^{i_{C}}=\left(i_{S}+i_{L}\right)$ or $i_{L} / i_{S}$ state. It is useful to operate at the lowest possible frequency for two reasons. First, the lowest $\omega$ maximizes $k$ in Eq. (2-10). This, in turn, maximizes the rate of change of $i_{L}$ in Eq. (2-9).* Therefore, the load current response is always at its time optimal. Second, lower frequencies produce the lowest number of converter switchings per unit time. Therefore, system switching losses are also minimized.

From the above discussions it is evident that ICB frequency control is an auxiliary control parameter which augments the effect of bang-bang phase control strategy. A system control strategy based on bang-bang phase modulation and the lowest safe frequency allowed by the instantaneous value of $\left(i_{S}+i_{L}\right)$ will provide the true (or exact) time optimal response in the load.

\subsection{Methods of Phase and Frequency Shifting}

Bang-bang control strategy requires frequent $180^{\circ}$ phase shifting between $90^{\circ}$ and $-90^{\circ}$. Similarly, frequency control requires frequency shifting. Both phase and frequency shifting are implemented by shortening or lengthening the time intervals between SCik switchings on one or both converters. Such switching interval perturbations can upset the capacitor voltage balance, if done randomly. A systematic way of altering the phase or frequency of the onephase ICB system without leaving voltage unbalances is presented here.

Frequency stifting is implemented through a series of modified switching intervals called transient switching sequence. The shortest transient switching sequence for phase shifting in the one-phase ICB is a three-step. If a

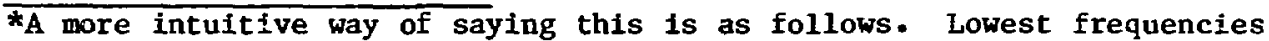
maintain highest capacitor voltages. Therefore, higher coil voltages are imposed which result in the fastest coil current response.
} 
phase shift corresponding to $\Delta t_{\phi}$ time shift between the storage and load converters is required, the three transient steps of the sequence are

$$
\begin{aligned}
& \Delta \mathrm{t}_{1}{ }^{(3)}=\Delta \mathrm{t}_{1}-\Delta \mathrm{t}_{\phi} / 2 \\
& \Delta \mathrm{i}_{2}{ }^{(3)}=\Delta \mathrm{t}_{1}-\Delta \mathrm{t}_{\phi} / 2 \\
& \Delta \mathrm{t}_{3}{ }^{(3)}=\Delta \mathrm{t}_{1}
\end{aligned}
$$

where $\Delta t_{1}{ }^{(3)}, \Delta t_{2}{ }^{(3)}$ and $\Delta t_{3}{ }^{(3)}$ symbolize the first, second, and third steps of a three-step sequence and $\Delta t_{1}$ is the steady-state switching time interval. Phase shifting can also be effected by transient steps which are lurger than the steady-state step. However, longer switching intervals produce temporary overvoltages on the capacitor. This may cause system over voltage failures if the ICB is running close to its minimum safe frequency. Therefore, transient switching steps which are longer than the steady-state step will not be considered further. Figure 10 shows an example of $0^{\circ}$ to $90^{\circ}$ phase advancing of the storage converter. Note that the shortened transient steps should always be applied to the converter that is advancing in time. Therefore, decreasing shifts in $\phi,\left(90^{\circ} \rightarrow-90^{\circ}\right)$ are implemented on the storage converter and vice versa." Examination of capacitor commutation voltage polarities in Fig. 10 shows ample voltage available during the transient. This suggests that a phase shift larger than $90^{\circ}$ could be implemented at once. Figure 11 shows one such example where $i_{L} / i_{S} \cong 0$ and a phase shift $\phi=90^{\circ}+$ $-90^{\circ}$ is implemented in three transient steps. Note that all commutation events have the correct capacitor voltage, $v_{C}$, polarity, albeit small during and after the shift. The conclusion is that if interval shortening transients are used, phase shifts to and from the threshold safe operating angles may be implemented in three steps. The safe threshold angles vs $i_{L} / i_{S}$ will be derived later in this report. The above conclusion is strikingly different from that of the three-phase ICB. The one-phase ICB not only has a simpler operating switching pattern, but it also has the much simpler three-step transient for bang-bang control implementation, whereas the three-phase ICB requires several more steps.

For conservative reasons it may be desired to Implement the $180^{\circ}$ shifts, dictated by bang-bang algorithm, In two three-step sequences. In this case 


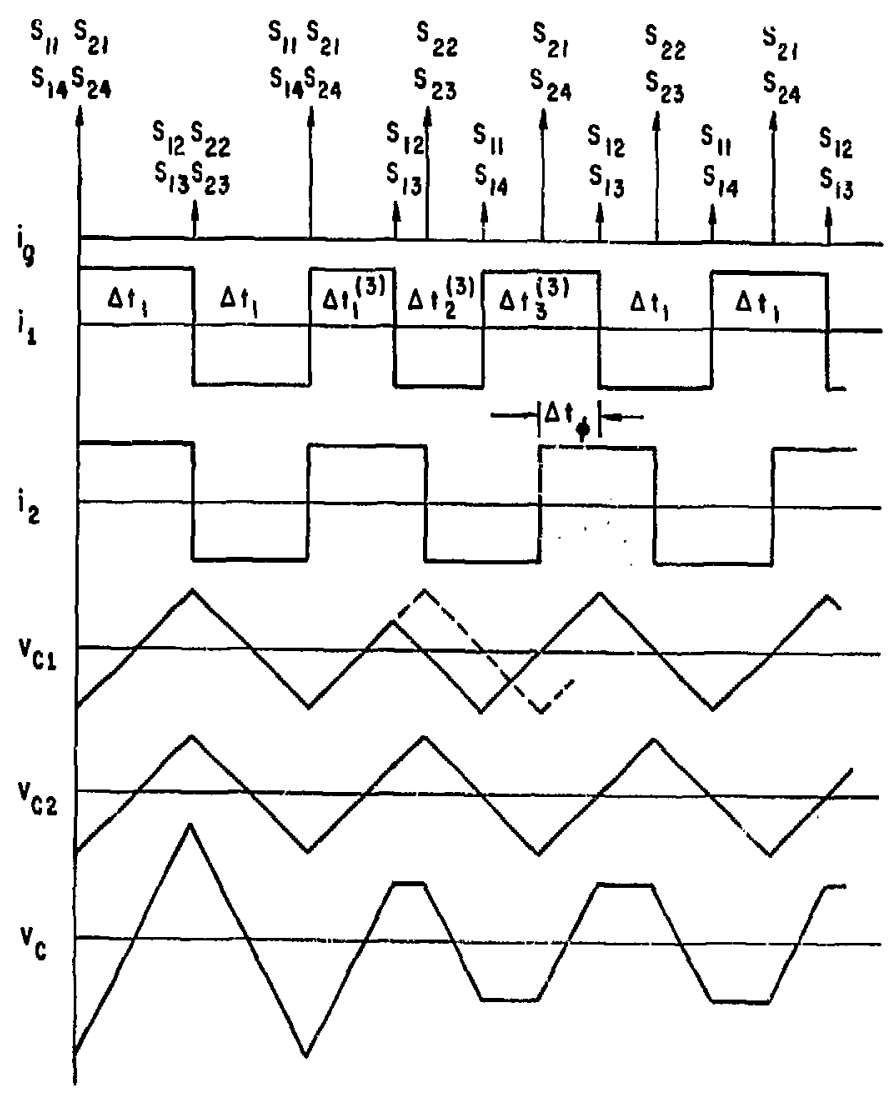

FIg. 10. Three-step cransient sequence phase shifting of $\phi=0^{\circ} \rightarrow-90^{\circ}$ at $1_{L} / i_{S}=1$.

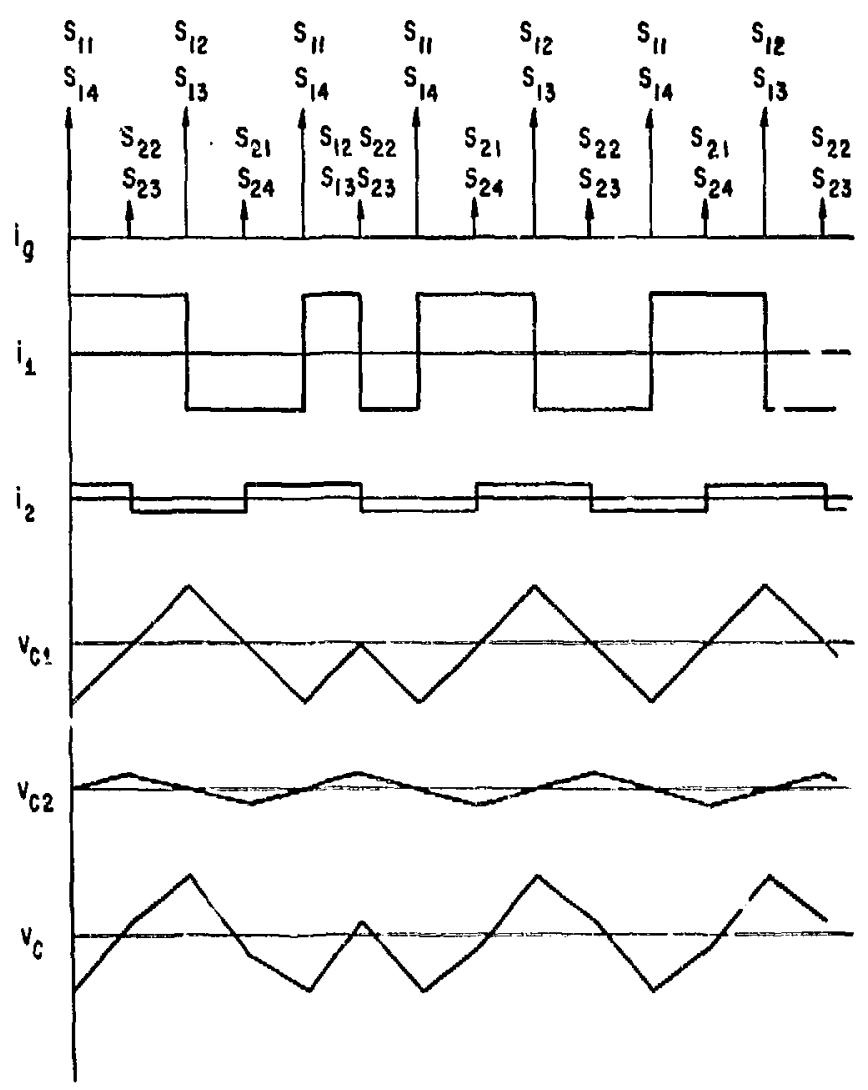

Fig. 11. Three-step transient sequence phase shifting of $\phi=90^{\circ} \rightarrow-90^{\circ}$ at $i_{L} / 1_{S} \cong 0$. 
the two sequences can obviously be implemented in tsolation from one another. For example the one-phase experiments presented in the next chapter were adapted from the three-phase transient sequence software with a minimum of modification. Thus, the three-step sequences of for example $90^{\circ} \rightarrow 0^{\circ}$ and $0^{\circ}+-90^{\circ}$ occur one switching interval apart. However, for time optimality, the bang-bang phase shifts should be implemented as quickly as possible. The transient steps of two three-step sequences may be merged to share one or two of their steps. In this case a composite flye-step or four-step sequence will result. For example, consider two three-step sequences intended to implement $\Delta t_{\phi}$ and $\Delta t_{\phi}^{\prime}$ time shifis, respectively:

\section{Sequence 1}

Sequence 2

$$
\begin{array}{lll}
\Delta t_{11}{ }^{(3)}=\Delta t_{1}-\Delta t_{\phi} / 2 & (2-20) & \Delta t_{12}(3)=\Delta t_{1}-\Delta t_{\phi} / 2 \\
\Delta t_{21}(3)=\Delta t_{1}-\Delta t_{\phi} / 2 & (2-21) & \Delta t_{22}(3)=\Delta t_{1}-\Delta t_{\phi}^{\prime} / 2 \\
\Delta t_{31}(3)=\Delta t_{1} & (2-22) & \Delta t_{32}(3)=\Delta t_{1} .
\end{array}
$$

The composite five-step transient sequence will be

$$
\begin{aligned}
& \Delta t_{1}\left(5 ;=\Delta t_{1}-\Delta t_{\phi} / 2\right. \\
& \Delta t_{2}(5)=\Delta t_{1}-\Delta t_{\phi} / 2 \\
& \Delta t_{3}(5)=\Delta t_{1}-\Delta t_{\phi}^{\prime} / 2 \\
& \Delta t_{4}(5)=\Delta t_{1}-\Delta t_{\phi}^{\prime} / 2 \\
& \Delta t_{5}(5)=\Delta t_{1} .
\end{aligned}
$$

The composite four-step transient sequence will be

$$
\begin{aligned}
& \Delta \mathrm{t}_{1}{ }^{(4)}=\Delta \mathrm{t}_{1}-\Delta \mathrm{t}_{\phi} / 2 \\
& \Delta \mathrm{t}_{i}(4)=\Delta \mathrm{t}_{1}-\Delta \mathrm{t}_{\phi} / 2-\Delta \mathrm{t}_{\phi}^{\prime} / 2
\end{aligned}
$$




$$
\begin{aligned}
& \Delta t_{3}(4)=\Delta t_{1}-\Delta t_{\phi}^{\prime / 2} \\
& \Delta t_{4}(4)=\Delta t_{1} .
\end{aligned}
$$

of course, for bang-bang control $\Delta t_{\phi}=\Delta t_{\phi}^{\prime}=T / 4$ or $90^{\circ}$. Further merging of the two sequences will produce a large phase shift three-step sequence which was discussed before. Note that if the total resulting phase shift is within the phase thresholds at the given $i_{L} / i_{S}$, then all of the above composite sequences will be safe and more conservative in commutation woltage than one three-step sequence.

The shortest transient switching sequence in one-phase ICB without lasting voltage unbalance, for frequency shifting, is a two-step.

If it is desired to shift from suitching interval $\Delta t_{1}$ to $\Delta t_{2}$, the two step sequence will be

$$
\begin{aligned}
& \Delta t_{1}{ }^{(2)}=\frac{l}{2}\left(\Delta t_{1}+\Delta t_{2}\right) \\
& \Delta t_{2}{ }^{(2)}=\Delta t_{2} .
\end{aligned}
$$

The transient step $\left.\Delta t_{1}: 2\right)$ is derived in Appendix 2. Figure 12 shows an example of a frequency shift upward and Fig. 13 shows an example for frequency shift downward. It is noted that the frequency shifting transient sequence is implemented on both converters as their switching times arrive. If the operating phase is other than zero, the above frequency shifting wiil substantially alter the phase relationship after the freque $: \because$ shift. Fo: example, Fig. 12 shows operation at $\phi=45^{\circ}$ price to the frequency shifi. The phase angle is changed to $\phi=90^{\circ}$, following the frequency transient sequar.ce. A similar effect is observed in Fig. 13 where the phase has been changed incdvertently from $\phi=90^{\circ}$ to $\phi=45^{\circ}$. Furthermore, the unintentional phase shift is dependent on which converter begins the frequency shifting transient first. For example, in Fig. 14 the ICB begins with the same initiai phase as in Fig. $12, \phi=45^{\circ}$, and goes through the same frequency shift. However, in this case the frequency transient sequence happens to begin on the storage converter first, as seen on the $\mathrm{v}_{\mathrm{Cl}}$ and $i_{1}$ traces. The resulting phase after the transient is sent to $\phi=-90^{\circ}$. 


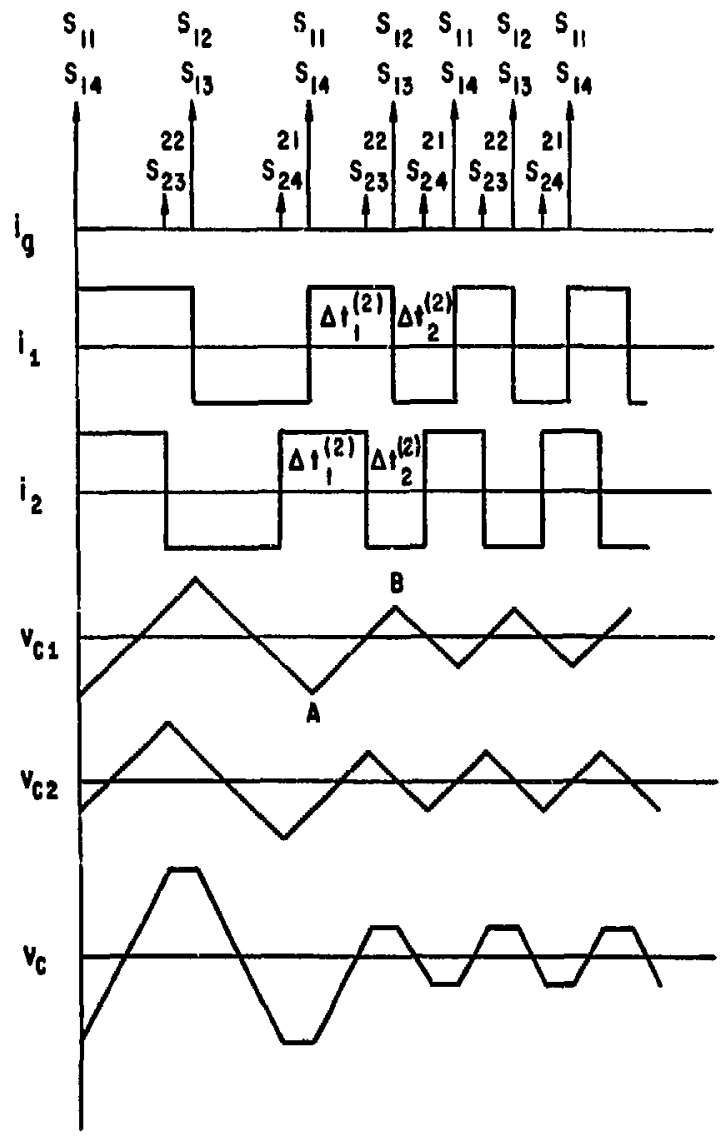

Fig. 12. Two-step transient sequence frequency shifting; frequency increase.

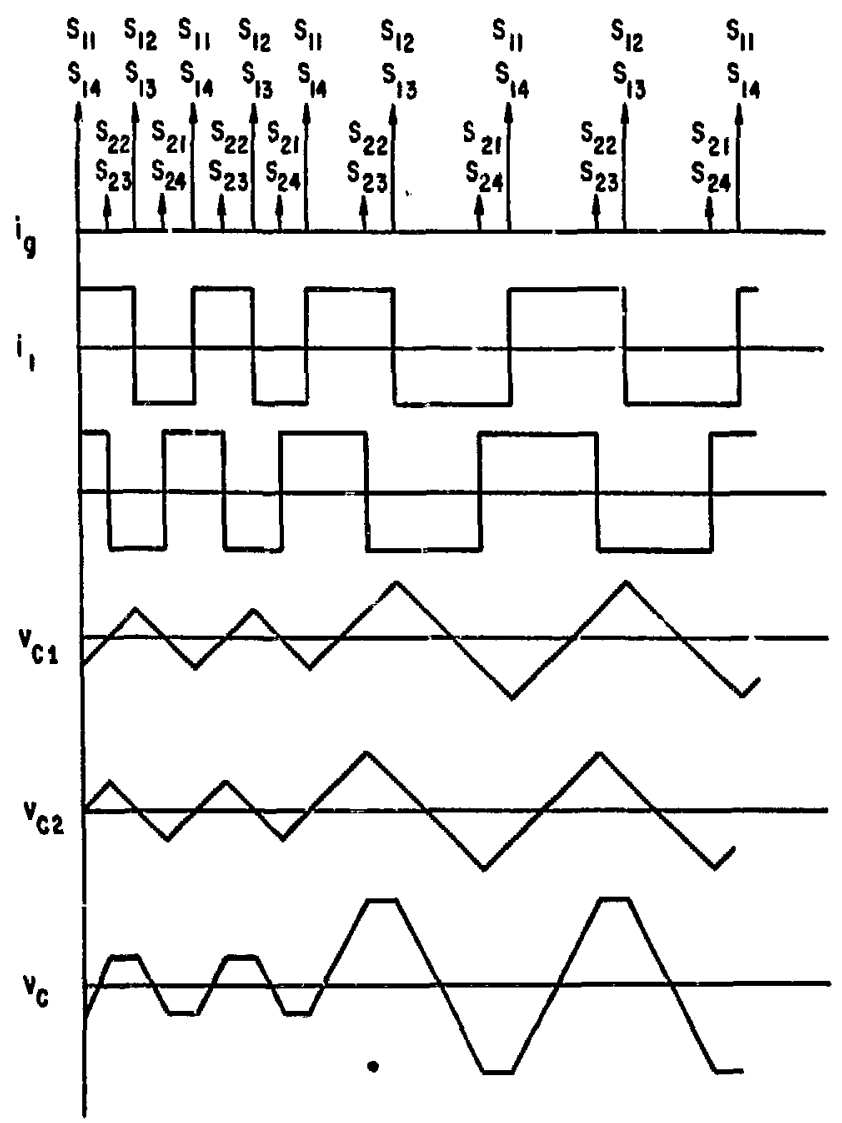

Fig. 13. Two-step transient sequency frequency shifting; frequency decrease. 


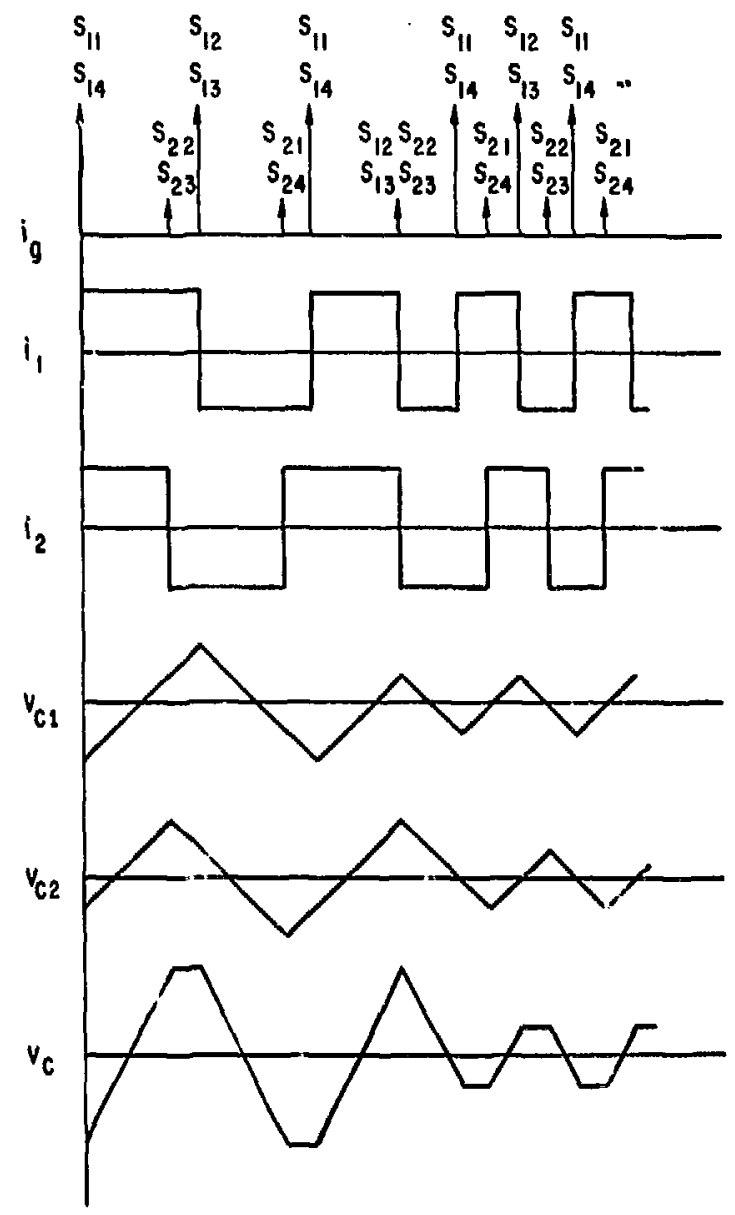

Fig. 14. Two-step transient sequence frequency shifting. Transient sequence began on the lagging converter. 
The unintentional phase shifting associated with frequency shifting can cause two difficulties. First, phase control comand is, at least temporarily, distorted. This will reduce the effectiveness of the phase controller. Second, and more importantly, the inadvertent phase shift may send the new phase outside the safe operating phase thresholds for the present $i_{L} / i_{S}$ state. Such commutation failures will not occur for the examples cited In Figs. 12, 13, and 14 because the initial phases were deliberately chosen to be low and the chosen state of $i_{L} / i_{S}=1$ is the least restrictive. However, if $i_{L} / i_{S} \cong=0$ and $\phi=90^{\circ}$ were the case in Fig. 12, the resulting phase, $\phi=180^{\circ}$, would cause a commutation fallure. Two techniques can be used to avoid the above difficulties. A simple techrigue is to always phase shift to zero prior to a frequency shift. Zero phase will survive a frequency transient sequence unchanged. Then, a phase shift can return the system to the desired operating phase. Slerging the phase shifting and frequency shifting transient sequences by one step, a composite transient sequence will result. Note that the initial phase shifting transient is implemented on the lagging converter, while the final phase shifting transient is implemented on the other converter to recover the original phase. Of course, the frequency shifting transient is implemented on both converters. Therefore, each converter will see a composite four step transient sequence as follows.

\section{Originally Lagging Converter}

$$
\begin{array}{lll}
\Delta t_{11}{ }^{(4)}=\Delta t_{1}-\Delta t_{\phi} / 2 & (2-37) & \Delta t_{12}(4)=1 / 2\left(\Delta t_{1}+\Delta t_{2}\right) \\
\Delta t_{21}{ }^{(4)}=\Delta t_{1}-\Delta t_{\phi} / 2 & (2-38) & \Delta t_{22}(4)=\Delta t_{2}-\Delta t_{\phi}^{\prime} / 2 \\
\Delta t_{31}{ }^{(4)}=1 / 2\left(\Delta t_{1}+\Delta t_{2}\right) & (2-39) & \Delta t_{32}(4)=\Delta t_{2}-\Delta t_{\phi}^{\prime} / 2 \\
\Delta t_{41}{ }^{(4)}=\Delta t_{2} & (2-40) & \Delta t_{42}(4)=\Delta t_{2}
\end{array}
$$

\section{Originally Leading Converter}

where

$$
\begin{aligned}
& \Delta t_{1}=\text { initial switching interval } \\
& \Delta t_{2}=\text { desired or final switching interval }
\end{aligned}
$$




$$
\begin{aligned}
& \Delta t_{\phi}=\text { the time shift required to bring the converters in phase } \\
& \Delta t_{\phi}^{\prime}=\text { the time shift required to implement the original phase at the } \\
& \text { new frequency. }
\end{aligned}
$$

Steps (2-39) and (2-41) are implemented sinultaneously on both converters. An example of this procedure is shom in FIg. 15 in which a $\phi=90^{\circ}$ is first shifted to $\phi=0$ and then back to $\phi=90^{\circ}$, following the frequency shift. Note that the phase shift-frequency shift-phase shift sequences do not have to be merged and may be implemented as separate sequences. Another technique for avoiding inadvertent phase shifts is to implement a totally merged phase and frequency shifting transient sequence such that as the frequexcy is being changed the phase is also being shifted by a correcting amount to compensate for the expected phase error. This technique requires that the frequency shifting sequence always start on eicher the leading or lagging converter so that the phase error can be calculated and compensated for. Since no particular advantage is observed in this method, it will not be discussed any further.

\subsection{Safe Operazing Limits}

As has been mentioned previously the capacitor in the one-phase ICB provides the voltage polarities necessary for commutating the SCR's of the two converters. For the current and voltage conventions shcwn on the circuit of FIg. 2, commutation from $s_{1}$ and $s_{4}$ to $s_{2}$ and $s_{3}$ in each converter requires $a$ positive capacitor voltage and vice versa. Depending on the state of current distribution in the two inductors, represented by $i_{L} / i_{S}$, and the phase difference between the two converters, such capacitor voltage polarities may not be available at the time of commutations. An example of this is shown in Fig. 16. Here, $i_{L} / i_{S} \cong 0.25$ and a phase shift from $\phi \approx 0^{\circ}$ to $\phi=135^{\circ}$ is attempted. The switching commands marked by $(x)$ on the $i_{g}$ trace will fail because of the wrong capacitor voltage polarity. In reality, the current and voltage waveforms will not proceed as shown after the first indicated commutation failure.

The thresholds of safe operating phases can be calculated as a function of current distribution state, $i_{L} / i_{S}$. The details of this calculation are 


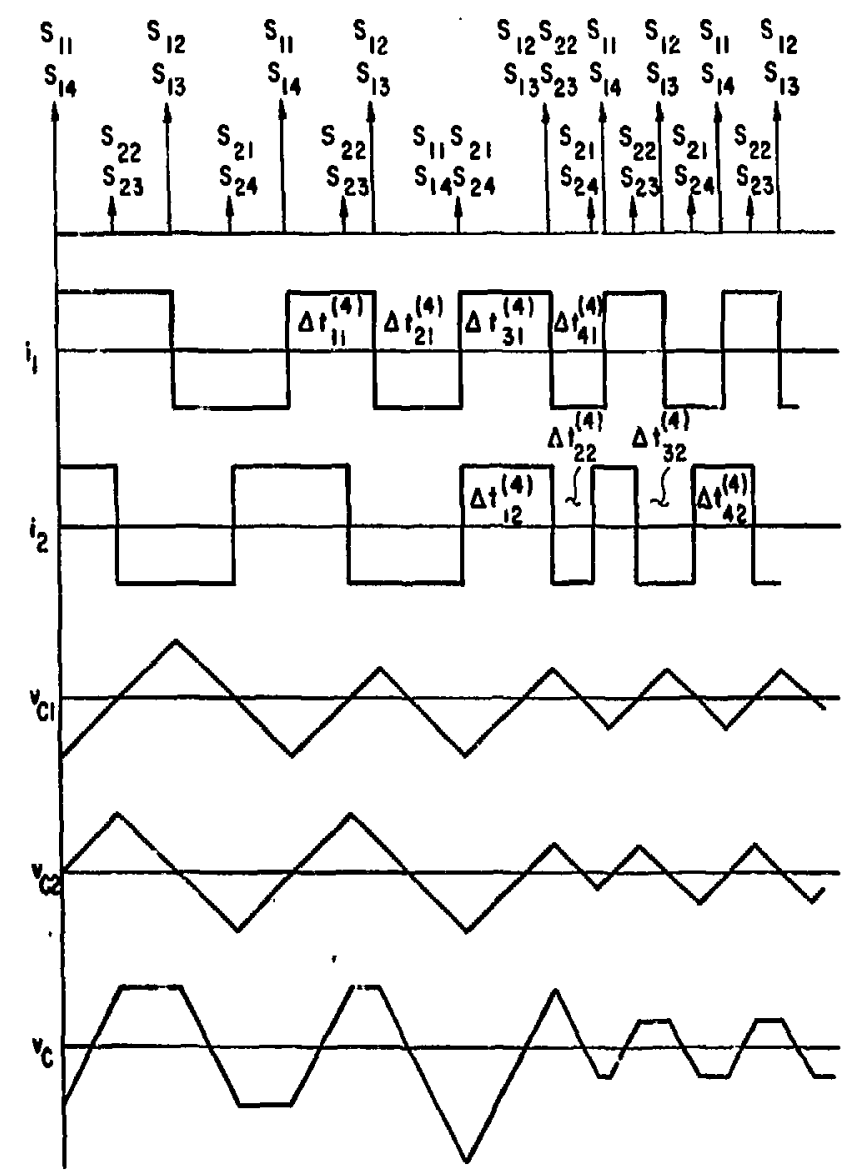

Fig. 15. Double four-step composite phase-frequency shifting to maintain constant rhase.

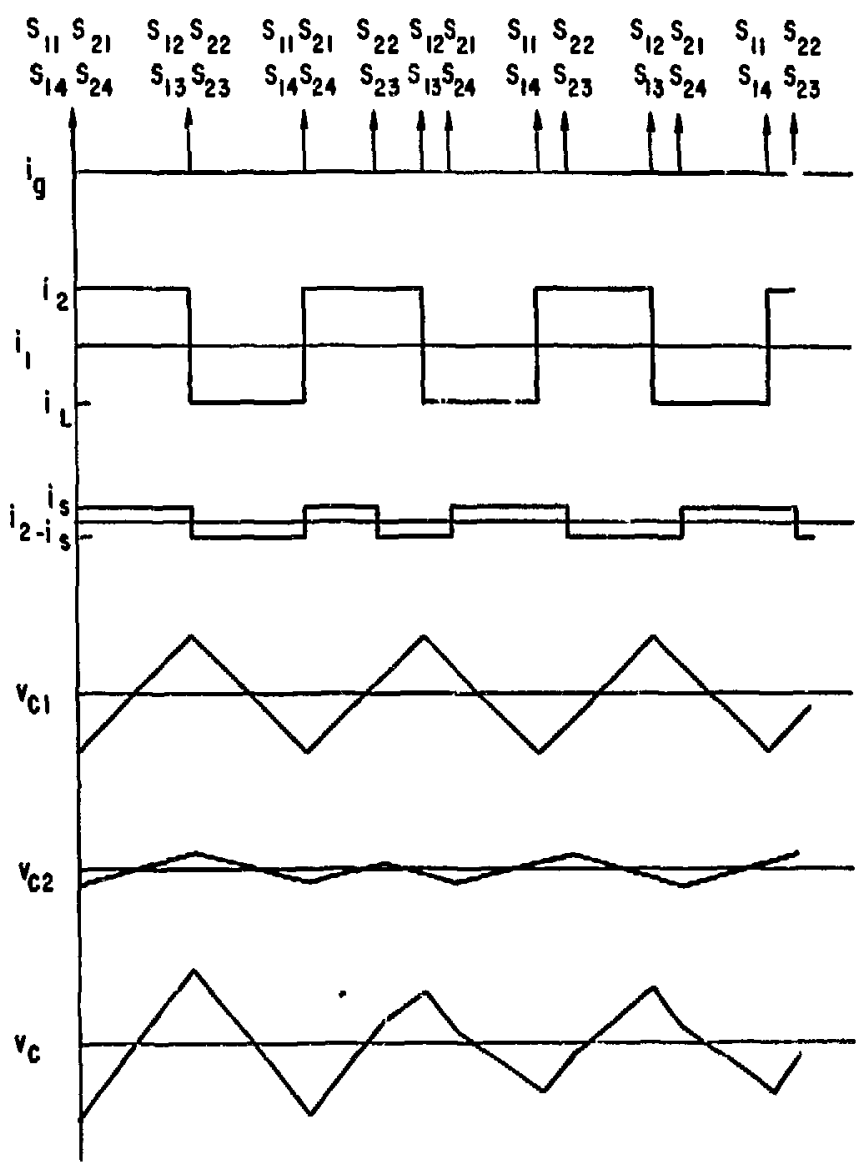

Fig. 16. Example of incorrect capacitor commutation vollage due to excessive phase difference. 
supplied in Appendix 3. The calculation shows that the ICB operating phase must obey the following inequalities.

$$
\begin{aligned}
& \frac{\phi}{90}<\frac{i_{L}}{i_{S}}+1 \\
& \frac{\phi}{90}>-\left(\frac{i_{L}}{i_{S}}+1\right)
\end{aligned}
$$

A plot of the above threshold lines is shown in Fig. 17. Operation anywhere within the cross hatched zone in this figure will be possible. Note that the most severe restriction $\left(|\phi|<90^{\circ}\right)$ exists at the beginning of the energy transfer when $i_{L} / i_{S}=0$. This implies that, in theory, bang-bang control strategy will not be able to start the energy trarifer due to commutation failures. In reality, bang-bang control will work after a brief transient period as will be shown in the next chapter. Although the plot of Fig. 17 gives the phase operating zone up to $i_{L} / i_{S}=1$, the information for the rest of the current distribution states $\left(i_{L} / i_{S}>1\right)$ can also be extracted from this plot as follows. For $i_{L} / i_{S}=2$, as an example, one may imagine the roles of the storage and load coils are swapped. Thus, a given $\phi^{\circ}$ operation at $i_{L} / i_{S}=$ 2 can be imagined as a $-\phi^{\circ}$ operation at " $i_{L} " /{ }^{i} S "=0.5$, which is on the graph of Fig. 17. Therefore, questions on operating phases $\phi$ at $i_{L} / i_{S}>1$ can be answered by their equivalent questions on operating phases $-\phi$ at $i_{S} / i_{L}<1$, as is shown in parentheses in Fig. 17. The above discussion indicates that the phases of bang-bang control strategy $\left(\phi=90^{\circ}\right)$ approach the thresholds of safe operation at the beginning and the end of a complete energy iransfer cycle. 


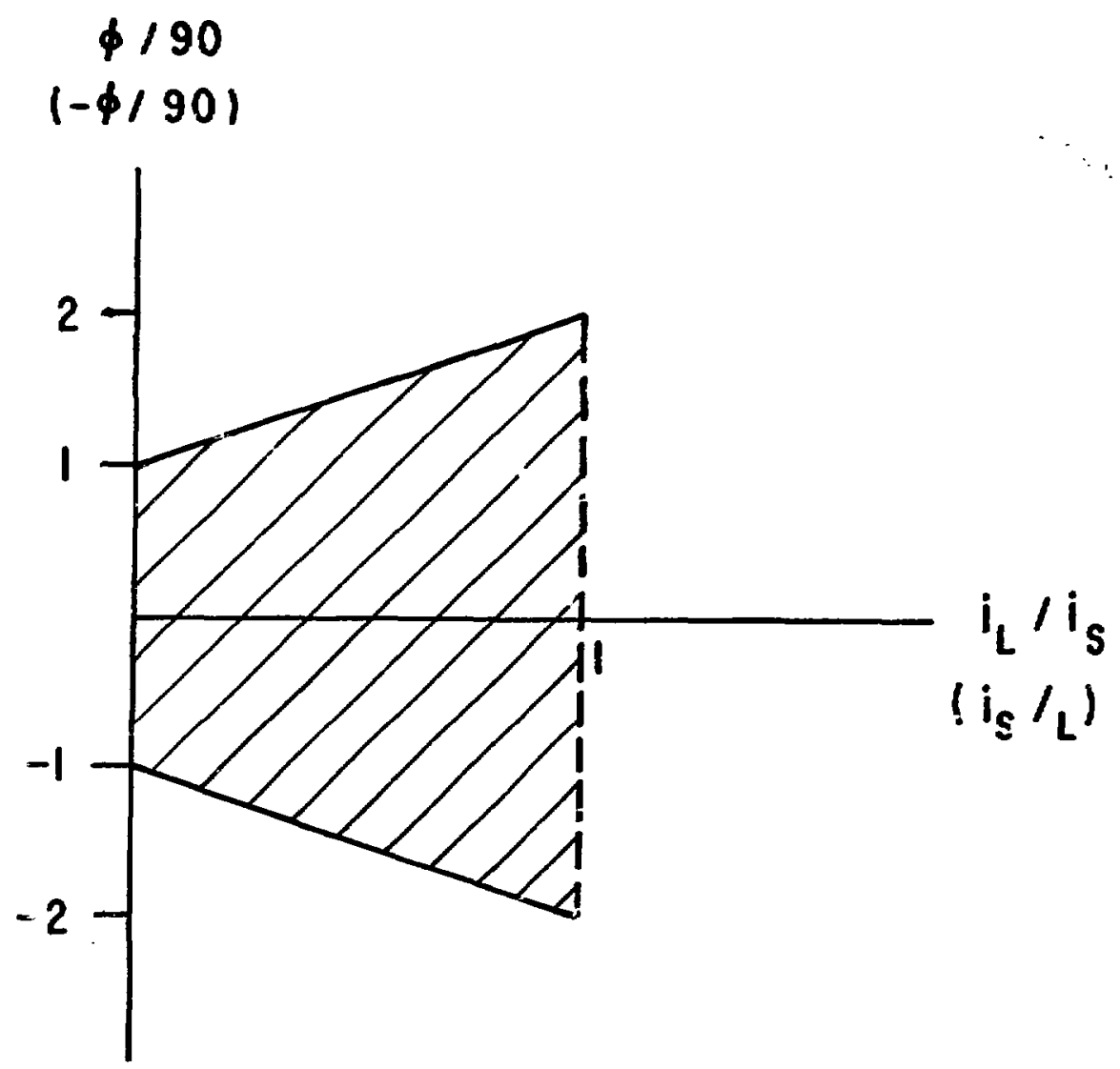

Fig. 17. Plot of acceptable operating phase thresholds in one-phase ICB. 


\section{EXPERIMENTAL RESULTS}

A one-phase ICB experiment was set up to verify the operation and phase shifting methods described in this report. The one-phase ICE was adapted from the three-phase prototype ICB system already built by Argonne National

Laboratory. (10) The existence of the superconducting coils, storage and load side three-phase converters and the operating and phase shifting microcomputer routines made the one-phase operation possible with minor modifications described below. The basic three-phase ICB circuit is shown in Fig. 18. The resistance paths in this circuit are for startup which will be discussed later. The switching sequence on each converter of this system is $s_{1} s_{5}$, $s_{1}, s_{6}, s_{2} s_{6}, s_{2} s_{4}, s_{3} s_{4}, s_{3} s_{5}, s_{1} s_{5} \ldots ., S C R^{\prime} s s_{2}$ and $s_{5}$ in each converter were completely inactivated by disconnecting their gates. $S_{C R} s S_{1}$ and $S_{6}$ were fed the gate pulses of $s_{1}$, and SCR's $S_{3}$ and $s_{4}$ were fed the gate pulses of $S_{4}$, on each respective converter. Thus, each converter was modified to a one-phase with the following gating sequence, $s_{1} s_{6}, s_{1} s_{6}, s_{2} s_{3}, s_{2} s_{3}$. It is seen that each pair of SCR's on each converter received a redundant firing pulse, $60^{\circ}$ Into its $180^{\circ}$ conduction. These spurious gating pulses were of no consequence since the first pulse would start the conduction. Effectively, a one-phase converter switching pattern was devised in which the sequence $S_{1} s_{6}$, $s_{3} s_{4}, s_{1} s_{6} \ldots$ acted as the sequence $s_{1} s_{4}, s_{2} s_{3}, s_{1} s_{4} \ldots$ of the previous chapter. With the above modifications the constant phase microcomputer operating software can run the one-phase bridge with identical phase calibration.

The phase shifting algorithm of the existing three-phase ICB used a fourstep transient switching sequence per $90^{\circ}$ shift as follows.

$$
\begin{aligned}
& \Delta t_{1}(4)=\Delta t_{1}-\Delta t_{\phi} / 2 \\
& \Delta t_{2}(4)=\Delta t_{1} \\
& \Delta t_{3}(4)=\Delta t_{1} \\
& \Delta t_{4}(4)=\Delta t_{1}-\Delta t_{\phi} / 2 .
\end{aligned}
$$

where $t_{\phi}$ is the time equivalent of the desired phase shift. With the gating alterations the first three steps of the above sequence are in the first step 
30

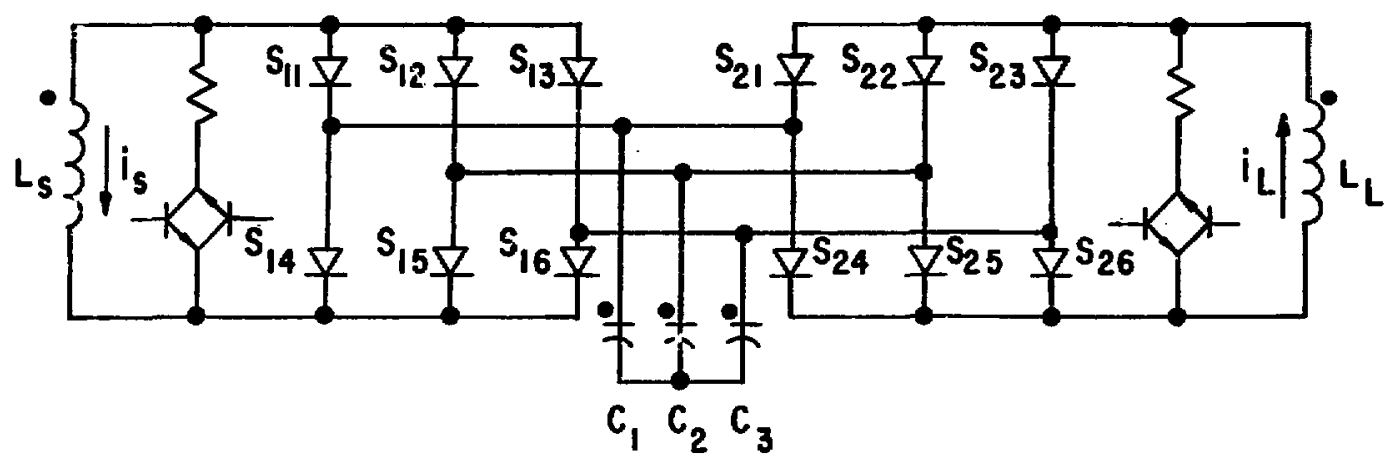

Fig. 18. Circuit diagram for the three-phase ICB. 
of the one-phase transient sequence and the fourth step, along with the next two steady state intervals, are in the second step of the one-phase transient sequence. This concept is restated below.

\section{One-Phase Steps}

$\Delta t_{1}(3)$
$\Delta t_{2}(3)$
$\Delta t_{3}(3)$

\section{Three-Phase Steps}

$$
\begin{aligned}
& \left\{\begin{array}{l}
\Delta t_{1}-\Delta t_{\phi} / 2 \\
\Delta t_{1} \\
\Delta t_{1}
\end{array}\right. \\
& \left\{\begin{array}{l}
\Delta t_{1}-\Delta t_{\phi} / 2 \\
\Delta t_{1} \\
\Delta t_{1}
\end{array}\right.
\end{aligned}
$$

$\left\{\begin{array}{l}\Delta \mathrm{t}_{1} \\ \Delta \mathrm{t}_{1}\end{array}\right.$

The one-phase transient steps $\Delta t_{1}{ }^{(3)}, \Delta t_{2}{ }^{(3)}$, and $\Delta t_{3}{ }^{(3)}$ are those defined for the one-phase ICB in the previous chapter. Therefore, the three-phase phase shifting algorithm, in effect, implements the three-step phase shifting algorithm for the one-phase ICB:

$$
\begin{aligned}
& \Delta t_{1}{ }^{(3)}=\Delta t_{1}{ }^{\prime}-\Delta t_{\phi} / 2 \\
& \Delta t_{2}{ }^{(3)}=\Delta t_{1}{ }^{\prime}-\Delta t_{\phi} / 2 \\
& \Delta t_{3}{ }^{(3)}=\Delta t_{1},
\end{aligned}
$$

where

$$
\Delta \mathrm{t}_{1}^{\prime}=3 \Delta \mathrm{t}_{1}
$$

The bang-bang control phase shift from $90^{\circ}$ to $-90^{\circ}$ and vice versa was implemented in two $90^{\circ}$ shifts in the three-phase ICB software. Therefore, the bang-bang control $180^{\circ}$ shifts in the one-phase ICB were also implemented in two three-step transient sequences of $90^{\circ}$ each. Figure 19 shows an example of such two segmented $180^{\circ}$ phase shift. From tue discussions of the previous 
$\begin{array}{lllllllllllll}s_{11} & s_{12} & s_{11} & s_{12} & s_{11} s_{21} & s_{11} & s_{22} & s_{21} & s_{22} & s_{21} & s_{22} & s_{21} & s_{22}\end{array}$ $\begin{array}{llllllllllllllllllllll}s_{14} & s_{13} & s_{14} & s_{13} & s_{14} s_{23} & s_{24} & s_{23} & s_{24} & s_{23} & s_{24} & s_{23} & s_{24} & s_{23}\end{array}$ $i_{9} i_{1}$

$i_{2}$
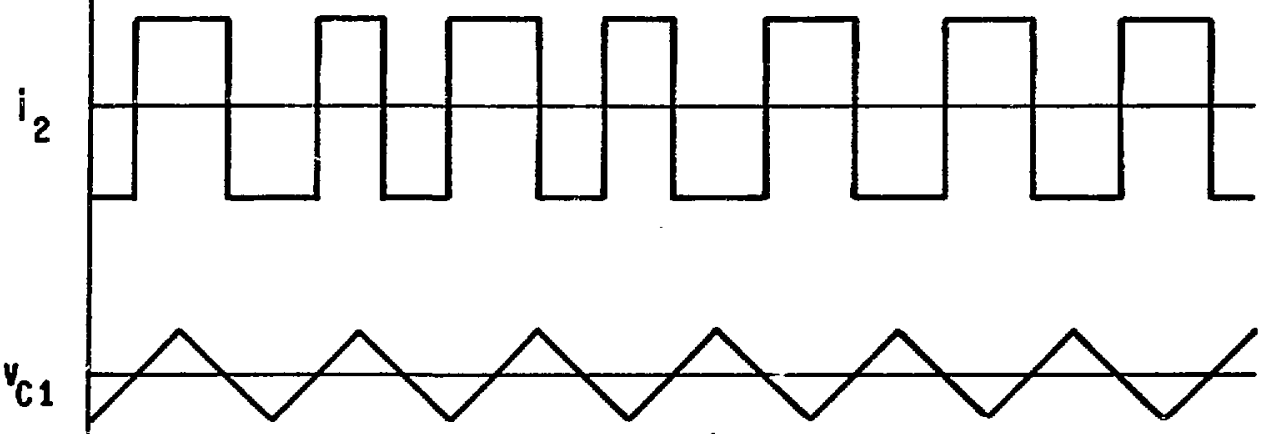

c2

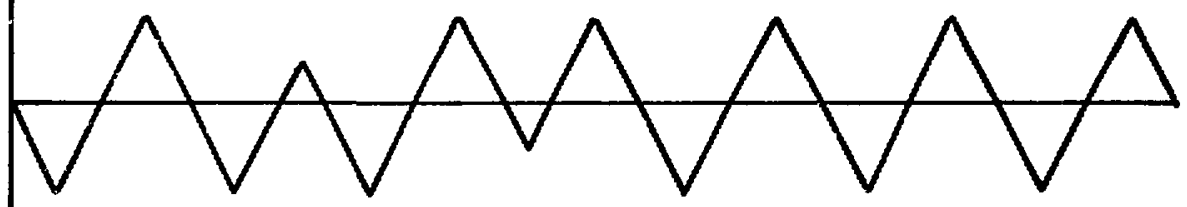

$v_{c}$

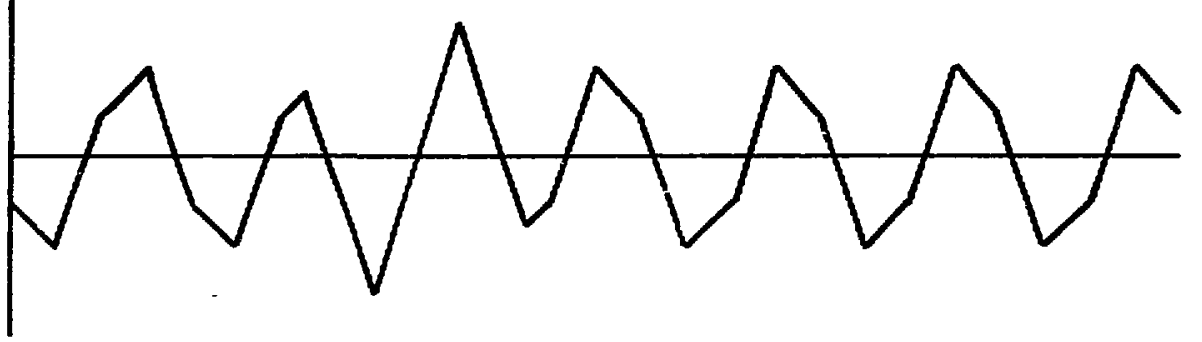

Fig. 19. Example of $180^{\circ}$ phase shift in two $90^{\circ}$ segments $\left(-90^{\circ} \rightarrow 0 \rightarrow 90^{\circ}\right)$. 
chapter it should be obvious that such a phase shift method is not the fastest transieut shifting in one-phase ICB.*

The circuit was designed to start operation from $s_{1} s_{5}$ in the three-phase ICB. This meant that the altered one-phase ICB would start from $s_{1} s_{6}$. Therefore, the two capacitors $C_{1}$ and $\mathcal{C}_{3}$ were precharged to a negative and positive voltage, respectively, of $1 / 2$ co with the dot indicating the positive voltage convention in Fig. 18. The series capacitors $C_{1}$ and $C_{3}$ constituted the equivalent capacitance shown in Fig. 2.

The resistor and antiparallel transistor paths, shown in Fig. 18, are necessary for the current startup of their respective coils. For example, without the load resistor the large inductance of the load coil will prevent the SCR holding current from being established in the coil, by the time the first SCR pair gate pulses are terminated. This will cause the first SCR pair to turn off upon the termination of the gate pulse. Therefore, the residual coil current will be interrupted. Similar discontinuous surges of current will be put in the load coil in each subsequent SCR pair firing. Therefors, the load coil current will never build up and energy transfer will noi occur. However, when the transistor switches place the resistor in parallel with the load coil SCR holding current vill be established in this resistor which is in series with the two gated SCR's and the ICB capacitor. Therefore, a net voltage can be established on this resistor jepending on the $c_{i}$ 'rating phase. This net voltage will eventually establish sufficient current in the load inductor to maintain SCR conduction after gating. At this point the transistor switches disconnect the lossy resistor from the circuit.

Some of the important test circuit values are listed in Table 3-1. A more complete listing appears in Reference 9.

\subsection{Constant Phase Operation}

An open loop constant phase $\left(\phi=60^{\circ}\right)$ test of the one-phase ICB was attempted to establish the viability of the circuit. The system operated successfully throughout a full energy transfer cycle from storage to load

\footnotetext{
$\overline{\text { *Proo } \bar{y} \text { of principle of }}$ one-phase ICB operation and control experiments could be done with the shortest preparation time by this choice.
} 
Table 3-1

$\mathrm{L}_{\mathrm{S}}=4 \mathrm{H}$
$\mathrm{L}_{\mathrm{L}}=4 \mathrm{H}$
Conve :er frequency $=\mathrm{F}=992 \mathrm{~Hz}$
Phase capacitance $=\mathrm{C}=2.5 \times 10^{-4} \mathrm{~F}$
SCR holding current $=I_{\mathrm{h}}=0.8 \mathrm{~A}$
Initial storage coil current $=i_{\mathrm{SO}}=100 \mathrm{~A}$
Initial capacitoz voltage $=v_{\mathrm{CO}}=220 \mathrm{~V}$

coil. Figure 20 shows the coil current waveforms through the trangfer cycle. The (t) mark at the left shows the zero time and zero current point for the storage current which is falling. The rising load current starts from the same time zero but is offset on the scope by about one division. It is observed that the currents follow a quarter cycle of a sinusoid which can be derived from Equations (2-8) and (2-9), evaluated with the circuit values. Figure 21 shows the capacitor voltage during the first few cycles of operation. This voltage was taken from one of the two series capacitors $\left(c_{1}\right)$ in the adapted one-phase ICB. The one-half precharge voltage of $-110 \mathrm{~V}$ and storage dominated waveform predicted in Fig. 3 is evident. The small transients at the switching points show the effect of commutation loop. currents, which is present in every capcitor voltage trace. Figures 22, 23, and 24 show the capacitor and coil voltages at three different spots during the energy transfer. The traces from top to bottom, respectively, are $v_{S},{ }^{\prime} C$, and $v_{L}$. The (+) mark at the left in every case shows the zero volt level of $\mathbf{v}_{\mathrm{S}}$. These waveforms are in close agreement with the prediction. For example, Fig. 23 shows the waveforms near $i_{S}=i_{L}$ which is the same behavior as in Fig. 4. The constant phase full energy transfer experiment was repeated at $\phi=$ $90^{\circ}$. As was shown in the previous chapter, $\phi=90^{\circ}$ is on the threshold of commutation at the beginning of the cycle, $i_{L} / i_{S}=0$. However, there is also no current in $L_{L}$ to be commutated. As the net $v_{L}$ voltage, shown in Fig. 3 , establishes some $i_{L}$ current, tine threshold phase goes beyond $90^{\circ}$ and $\phi=90^{\circ}$ commutation progressively improves. These arguments were supported iy the 


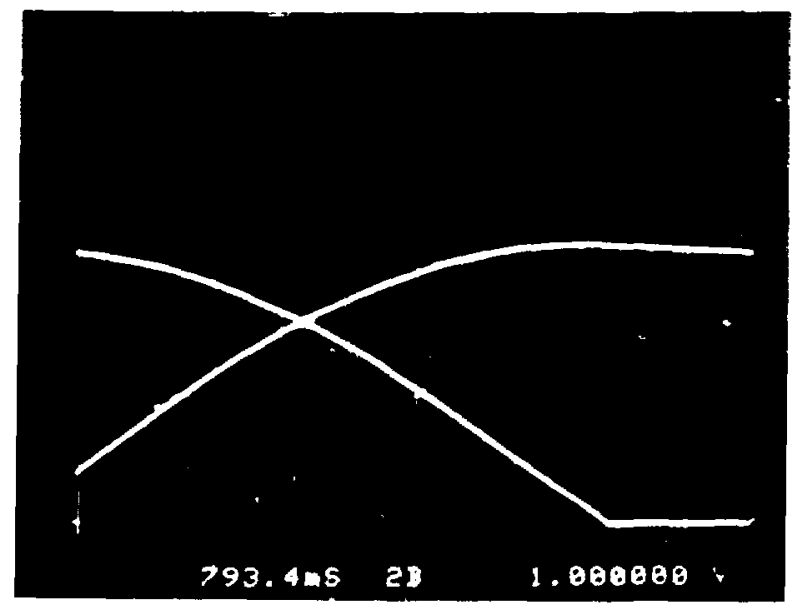

Fig. 20.

Coil current waveforms through a full energy transfer at $\phi=$ $60^{\circ}$. Scale: $25 \mathrm{~A} / \mathrm{div}$.

Fig. 21.

Capacitor voltage during the first cycles of operation. Scale: $50 \mathrm{~V} / \mathrm{div}$.
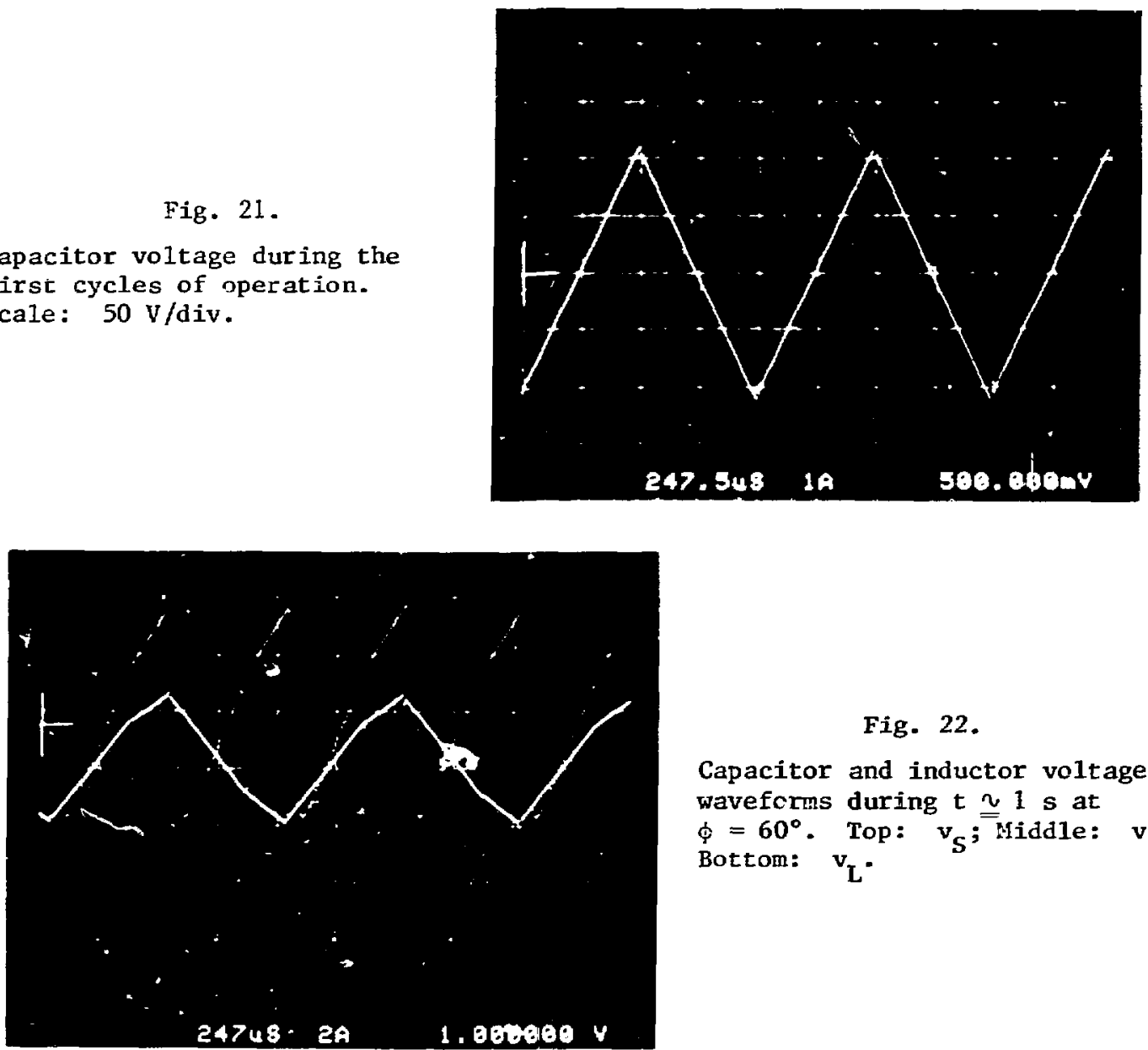

Fig. 22.

Capacitor and inductor voltage wavefcrms during $t \cong 1$ s at $\Phi=60^{\circ}$. Top: $\mathrm{v}_{\mathrm{S}}$; Middle: $\mathrm{v}_{\mathrm{C}}$; Bottom: $\mathbf{v}_{\mathbf{L}}$. 


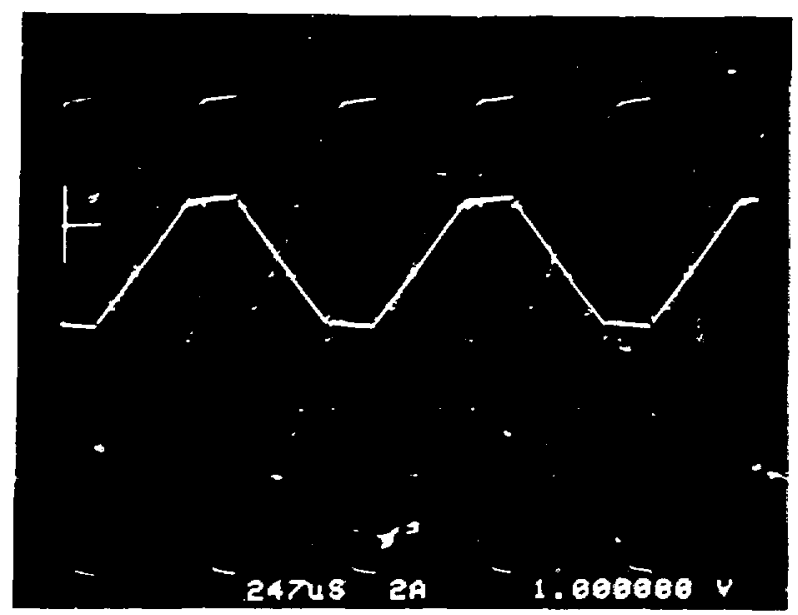

Fig. 23.

Capacitor anl i-juctor voltage waveforms during $t \cong 2.5$ s at $\phi=60^{\circ}$. Top: vs; Middle: $v_{0}$; Bottom: $v_{L}$.

Fig. 24 .

Capacitor and inductor voltage waveforms during $t \bumpeq 4 s$ at $\phi=$ $60^{\circ}$. Top: $v_{S}$; Midd $1 e: v_{C}$; Bottom: $\mathrm{v}_{\mathrm{L}}$.

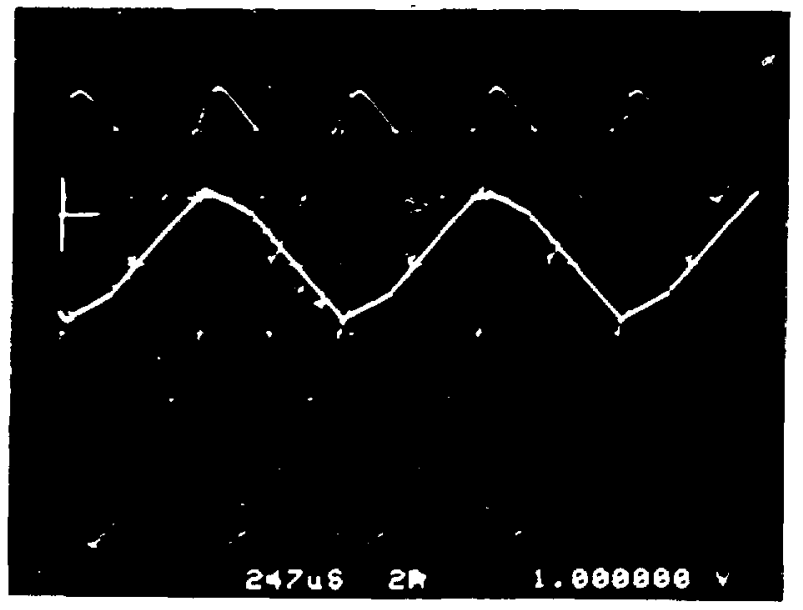


success of energy transfer at a constant $\phi=90^{\circ}$. Figure 25 shows an example of the inductor and capacitor voltages. The $\phi=90^{\circ}$ phase relationship in this oscillogram can be verified by the fact that the storage and $\operatorname{col} 1$ voltage switchings are occurring at equal intervals.

The three-step phase shifting algorithe was tested in a closed loop bangbang control experiment. The $180^{\circ}$ phise shifts were done in two three-step $90^{\circ}$ shift segments, as was shown in Fig. 19. Figure 26 shows an example of the capasitor vcltage during a $\phi=-90^{\circ} \pm 0 \dot{\psi}=90^{\circ}$ phase shift. For better understanding of how this waverorm is produced, Fig. 19 should be studied. The straight ise connecting the highest two capacitor voltage peaks shows the switching intervc in which the two converters are in phase $(\phi=0)$. Figure 27 shows the inductor voltages during the same phase shift as that in Fig. 26. The change of average load voltage from positive to negative can be seen as the load voltage oscillati uns (top trace) go through the shifting transient steps. A similar change of net storage voltage from regative to positive can be seen in the bottom trace. The switching interval with $\phi=0^{\circ}$ is again seen in both traces as a straight line. The zero voltage axis of each trace runs chrough the miapoint of this line.

The experimental evaluation of the one-phase ICB established for the first time the practicality of this system as a controllable two quadrant power supply for superconducting magnets. 


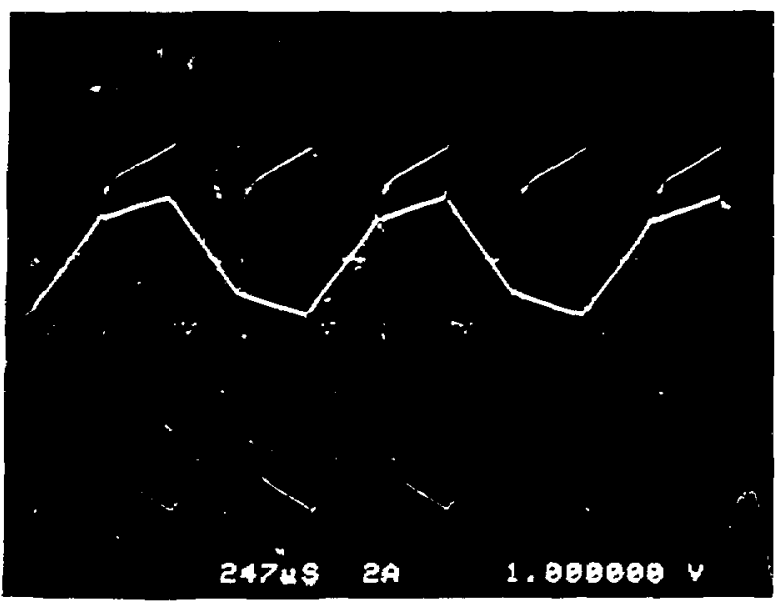

Fig. 25.

Capacitor and inductor voltage waveforms during $t \simeq 2 s$ at $\phi=$ $90^{\circ}$. Top: $v_{s}$; Middle: $v_{c}$; Bottom: $v_{L}$.

Fig. 26.

Capacitor voltage during a $\phi=$ $90^{\circ}$ to $-90^{\circ}$ phase shift.
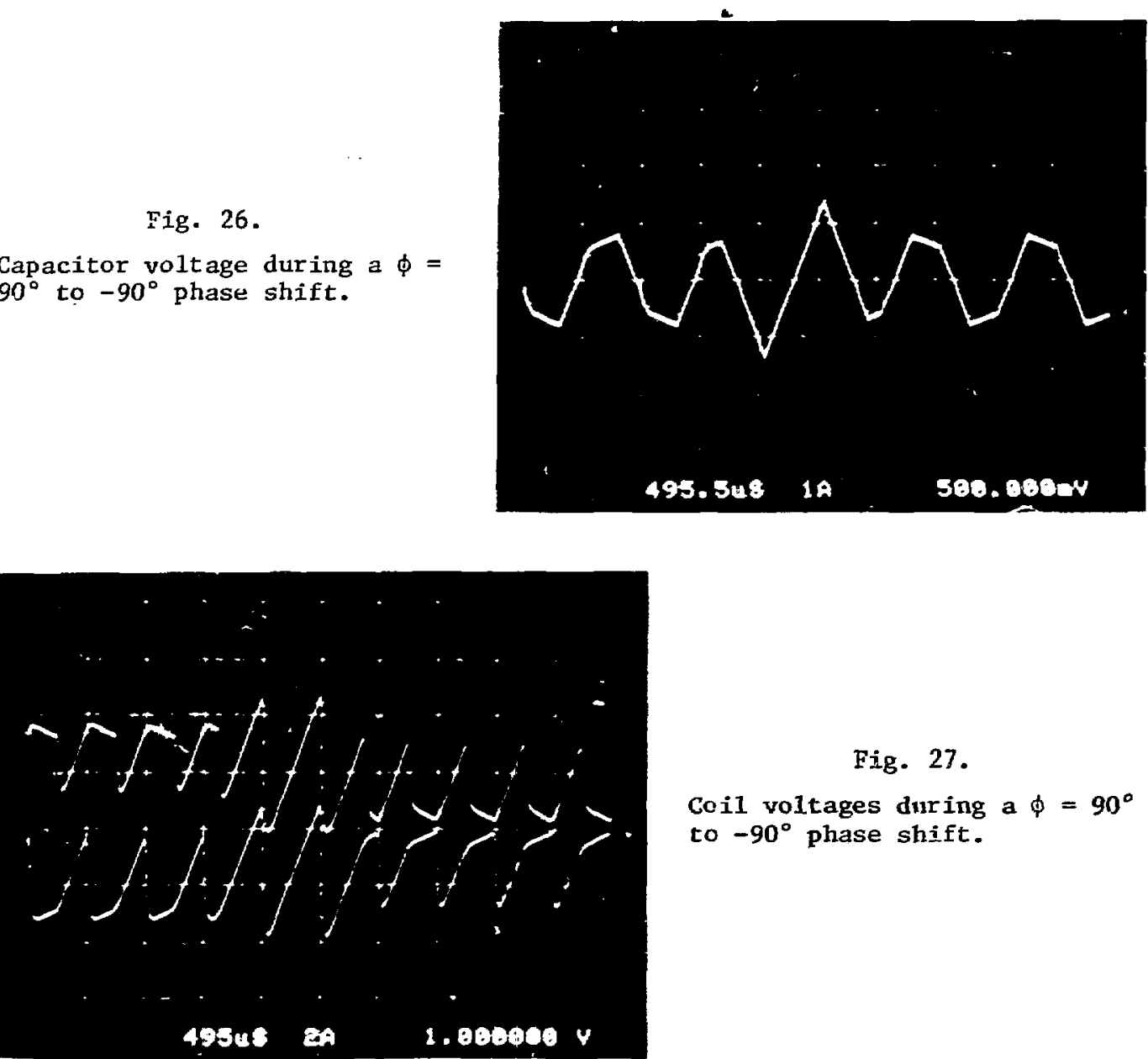

Fig. 27.

Coil voltages during a $\phi=90^{\circ}$ to $-90^{\circ}$ phase shift. 


\section{DISCUSSION AND CONCLUSTONS}

Several interesting Liansients wers observed at the beginning of the onephase ICB operation. The startup resistor, which was discussed previously, set up a switching transient on the load converter which is manifested on the load voltage shown in Fig. 28. This oscillogram is frow a constant phase experiment at $\phi=60^{\circ}$. The zero time and zero voltage point is marked by $(+)$ at the left of the frame. The dcwnward shift of the voliage wave envelope during the first $1 \mathrm{~ms}$ is due to the initial phase shift of $\phi=0^{\circ}$ to $\phi=60^{\circ}$ which the operating routine implenented in constant phase experiments. This first two-cycle converter phase shift is better observed in an expanded view of the first $2.5 \mathrm{~ms}$ of the operation shown in Fig. 29. The storage coil voltage is placed at the top of this oscillogram for timing reference. It is observed that the first two switchings, occurring at the positive peaks of the wave, are synchronized with those of the storage. Then phase shifting switching transients advance the load voltag. switching points by $60^{\circ}$. The downward trend of the load voltage envelope is also visible through this transient switching. Referring back to Fig. 28, the longer transient on the load voltage envelope is a result of coil inductor current builcing up. However, this fortuitous manifestation of load current buildup actualiy comes about from an unexpected mode of SCR operation described below. The startup resistor, which was designed for three-phase ICB operation at somewtat higher voltages, had too high a value for establishing latching SCR currents. Therefore, the load converter SCR's would stop efficient conduction shortly after the gate pulse terminated. The high impedance SCR conduction reduced some of the capacitor voltage swing from appearing on the load coil. This is seen in the lower amplitude oscillations at the beginning of the operation in Fig. 28. However, as the load coil current builds up toward the SCR holding current, $300 \mathrm{~mA}$, the SCR's conduct more efficiently and for a longer time following the gate pulse termination. Therefore, the capacitor voltage is more faithfully reflected on the load coil with increasing time. Finally, the resistor and load coil combination have enough current to sustain full SCR conduction through the switching interval and the expected steady state load voltage appears. Shortly after this the startup resistor is disconnected by the microcomputer. Note that the regative peaks of load voltage are at steajy atate level 


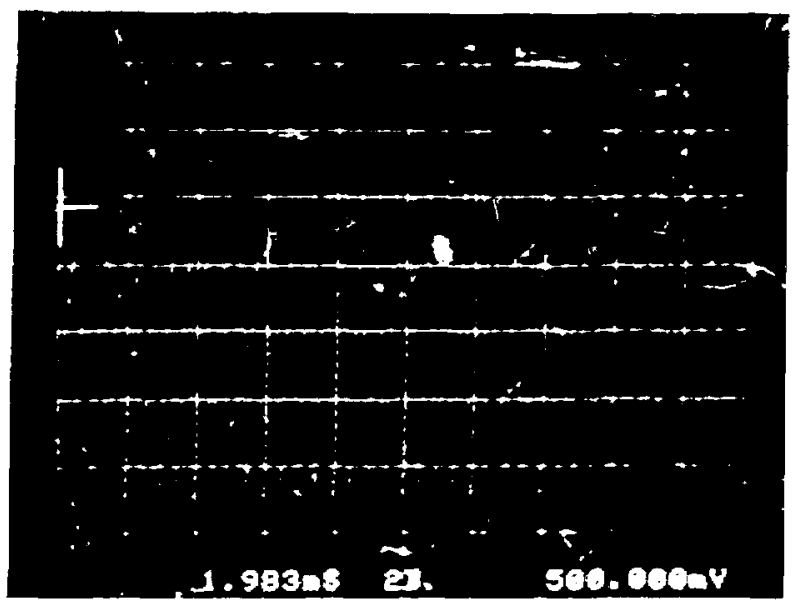

Fig. 28.

Load coil voltage startup transient while the load parallel resistor is on.

Fig. 29.

Inductor voltages at the beginning of operation showing the first phase shift transient on the load voltage. Top: storage voltage; Bottom: load voltage.

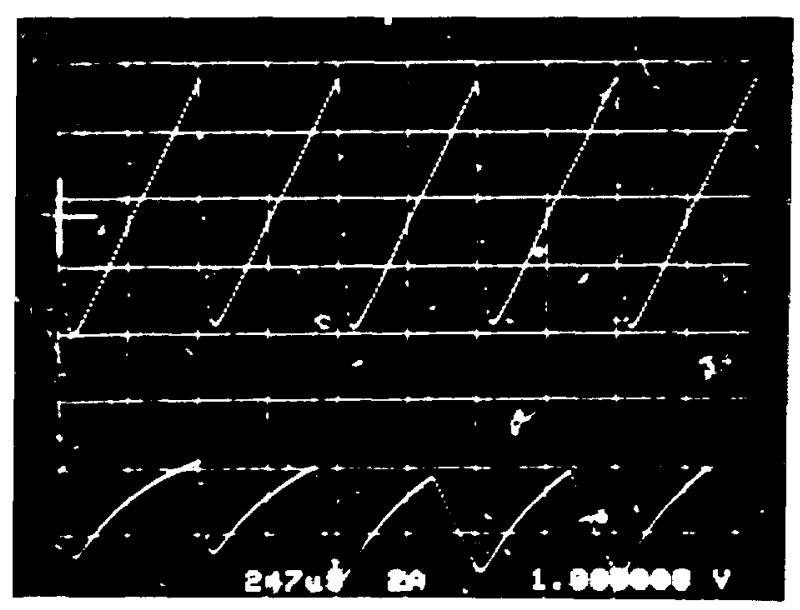


from the beginning of this transient.* This is because this part of the coll voltage cccurs when the SCR's are being pulsed and are conducting efficiently. The above discussion implies that had the startup resistor been chosen at a lower value, full SCR conduction would have started earlier in the load current buildup. However, the partial SCR conduction phenomenon presented an opportunity to see the load coil current rise toward SCR latching current.

The startup zesistor was observed to drop out in approximately $6 \mathrm{~ms}$ to 16 ms, depending on the operating phase and voltage levels involved.

Another transient in the ore-phase ICB is the decay of capacitor voltage unbalances. It was observed in the experiments that incorrect precharging of the capacitor was corrected within the first few cycles of converter operation. Incorrect capacitor precharging will produce a voltage offset as the storage converter begins to switch. This concept is dramatized in Fig. 30 . At $\phi=90^{\circ}$, as an example, the load converter will rectify the offset oscillating capacitor voltage. The high feak capacitor voltage half cycles will drive more current through the load coil and startup resistor, while the low peak half cycles will drive less current. This compensating discharge will eventually null the dc offset in the capacitor voltage since the offset is not being sustained by any source. The above process requires long gate pulses, extending almost the entire $180^{\circ} \mathrm{SCR}$ conduction interval. In our experiment the spurious second gate pulse on each $\mathrm{SCR}$, which was discussed before, can produce the same effect. However, single short pulse SCR firing will also tend to balance the capacitor voltage, but in a different transient conduction mode.

The experiments on the one-phase ICB established that this circuit is a practical contender for superconducting magnet power supply. I. addition, this circuit has certain advantages over the other multiphase ICB circuits. The most prominent advantage is the simplicity of the circuit and its control. Compared to the other ICB's this circuit has fewer sitches. From the circuit complexity point of view this can mean lower number of snubbers, gate drives, and similar switching and protection auxiliary equipment. From 


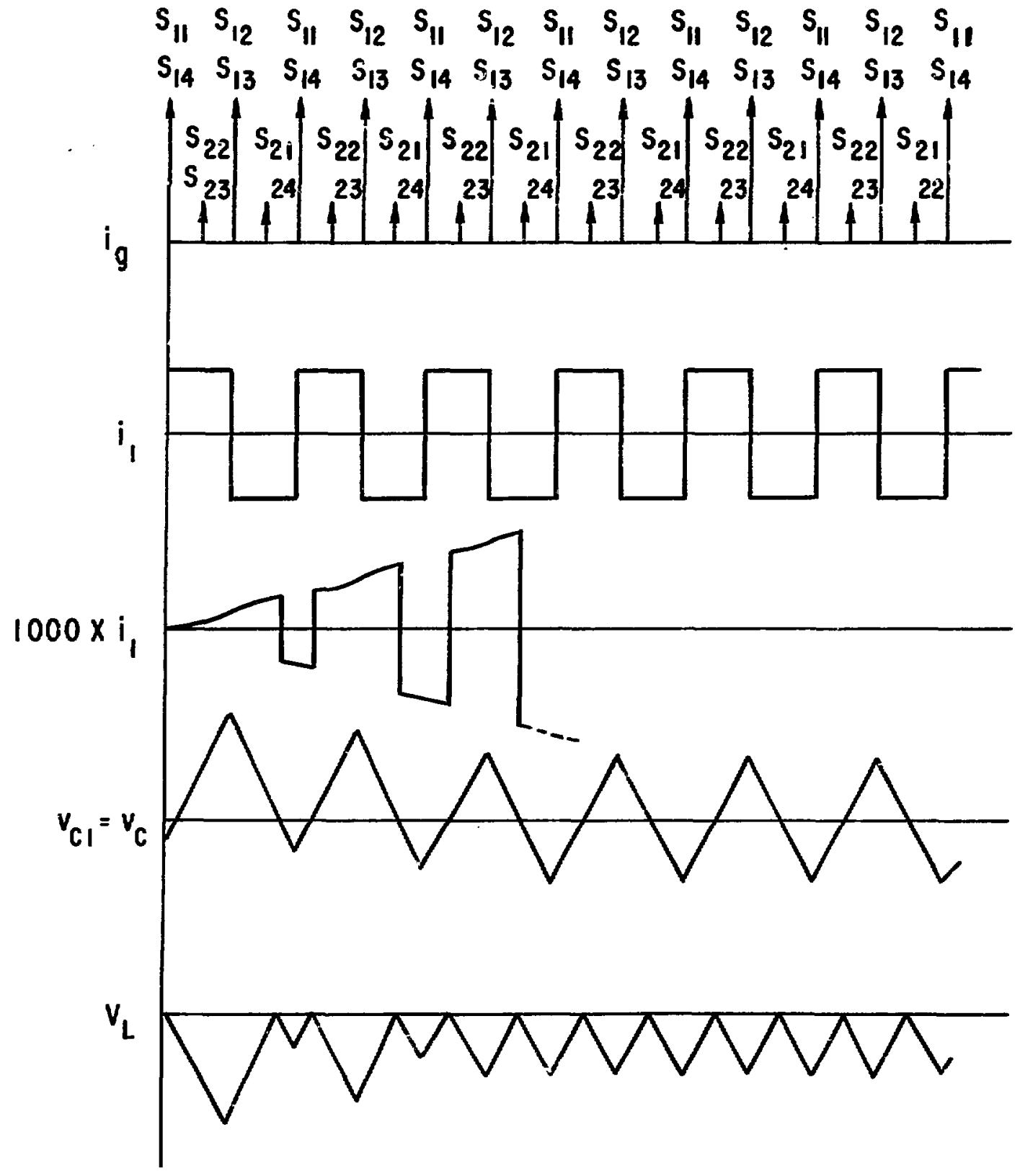

Fig. 30. An exaggerated example of capacitor voltage self balancing in the one-phase ICB. (Long SCR gate pulses starting from the $i_{g}$ impulse instants are assumed.) 
the control point of view, the one phase ICB has a binary switching pattern on each converter which is the simplest pattern to implement by software or discrete electronics. Furthermore, the phase and frequency shifting transient switching sequences of the one-phase ICB are simpler than the three-phase ICB. This also simplifies the control software and electronics. Another potentially significant feature of the one-phase ICB is that each converter switch utilization factor is $50 \%$ of the cycle, rather than, for example, $33 \%$ for the three-phase ICB. This could mean a substantial reduction in the total number of SCR's needed for the one-phase ICB which will translate to a large cost and complexity savings in a large system. Finally, the one-phase ICB proved to be a resilient circuit to voltage errors and commutation failures. This, coupled with the robust strategy of bang-bang control, $(9)$ is an attractive design option for magnet supplies.

For better understanding of the potential value of the unique features of the one-phase ICB, an in-depth comparative study of the one-phase and multiphase ICB circuits is required. Such a study will be undertaken by the authors in the future.

\section{ACKNOWLEDGEMENT}

The authors wish to express their appreciation for the valuable assistance of Professor Hidehiko Okada of Oiter University, Japan, Mr. Shirabe Akita of Central Research Institute of Electric Power Industry, Japan, and Mr. Masaru Hirano of University of Wisconsin, Madison in preparation and testing of the one-phase ICB. Also the support of Argonne National Laboratory and Applied Superconductivity Center (ASC) of University of Wisconsin, Madison, and particularly Professor R. W. Boom, Director of ASC is gratefully acknowledged. 


\section{REFERENCES}

1. "Tokamak Experimental Power Reactor Conceptual Design," Argonne National Laboratory Report ANL/CTR-76-3, 1976.

2. R. W. Boom, H. A. Peterson, et a1., Wisconsin Superconductive Energy Storage Project Report, Vol. I, July 1974.

3. F. E. Mills, "The Fermilab Cryogenic Energy Storage System," IEEE Trans. on Magnets, Vol. MAG-11, No. 2, March 1975, p. 489.

4. J. F. Haver and H. J. Boenig, "Electrical Tests of $30 \mathrm{MJ}$ Supezconducting Magnet Energy Storage System within the Bonneville Power Administration Power Grid," Los Alamos Natiunal Laboratory Report, LA-UR-83-2574, September 1983.

5. H. A. Peterson, N. Mohan, W. C. Young, and R. W. Boom, "Superconductive Inductor-Converter Units for Pulsed Power Loads," Proc. of Int. Conf. on Energy Storage, Compression, and Switching, Asti-Torino, Italy, November 1974 .

6. E. P. Dick and H. Dustman, "Inductive Energy Transfer using a Capacitor," Proc. of Int. Conf. on Energy Storage, Compression, and Switching, AstiTorino, Italy, November 1974.

7. R. L. Kustom, Thyristor Networks for the Transfer of Energy Between Superconducting Coils, University of Wisconsin Press, 1980.

8. H. L. Laquer, F. L. Ribe, and D. M. Wilson, "Energy Storage and Switching with Superconductors as a Power Source for Magnetic Fields in Pulsed Thermonuclear Experiments and Reactors," Intersociety Energy Conversion Engineering Conf., Boston, MA, August 1971.

9. M. Ehsani and R. L. Kustom, "Development, Analysis and Control of the Inductor-Converter Bridge," Argonne National Laboratory Report ANL/FPP/TM-144, 1981.

10. R. Fuja, R. L. Kustom, and R. P. Smith, "Resulte fror a Model System of Superconducting Solenoids and Phase Shifting Bridge fuc Pulsed Power Studies for Proposed Tokamak EF Coils," Cryogenic Engineering Conf., 1977. 


\section{APPENDIX 1}

\section{Calculation of Average Coll Power}

The current waveforms of the sources shown in Fig. 7 are square waves as shown in Fig. 4. The Fourier series expansion of these currents at an arbitrary relative phase angle, $\phi$, with $i_{1}$ as reference is

$$
\begin{aligned}
& i_{1}(t)=\sum_{n=1}^{\infty} B_{1} \text { sin } \omega t \\
& I_{2}(t)=\sum_{n=1}^{\infty} A_{2} \cos \omega t+B_{2} \sin \omega t
\end{aligned}
$$

where

$$
\begin{aligned}
& B_{1}=\frac{2 i}{n \pi}\left[1+(-1)^{n+1}\right] \\
& A_{2}=\frac{2 i_{L}}{n \pi}\left[1+(-1)^{n+1}\right] \sin n \phi \\
& B_{2}=\frac{2 I_{L}}{n \pi}\left[1+(-1)^{n+1}\right] \cos n \phi
\end{aligned}
$$

The capacitor voltage due to the currents $i_{1}$ and $i_{2}$ is

$$
v_{C}(t)=v_{C O}+\frac{1}{c} \int_{0}^{t}\left[i_{1}(t)+i_{2}(t)\right] d t,
$$

where $v_{C O}$ is the initial voltage on the capacitor. The instantaneous output power of $i_{1}$ source is

$$
p_{1}=i_{1} v_{C} \text {. }
$$

The average power over one $i_{1}$ source cycle is

$$
\left\langle p_{1}\right\rangle=\frac{1}{T} \int_{0}^{T} p_{1}(t) d t
$$


Substituting equations $(A-1-1)$ and $(A-1-2)$ in Equation $(A-1-6)$ and then substituting the result in equation $(A-1-7)$ and finally in Equation (A-1-8), will result in

$$
\left\langle p_{1}\right\rangle=\sum_{n=1}^{\infty} \frac{1}{2 n \omega c} B_{1} A_{2} .
$$

Now, substitution of Equations $(A-1-3)$ and $(A-1-4)$ into Equation (A-1-9) will result in

$$
\left\langle p_{1}\right\rangle=\sum_{n=1}^{\infty} \frac{4 i s^{i} L}{n^{3} \pi^{2} w c}\left[1-(-1)^{n}\right] \sin n \phi
$$

This $i_{1}$ source average power is the same as the storage converter output power shown in Equation (2-1).

The quadrometric method of calculating the average power avoids the infinite series used above by expressing $i_{1}$ and $i_{2}$ in terms of a switching function defined in Reference 9. The currents in terms of this function are

$$
\begin{aligned}
& i_{1}(t)=i_{S} S q \frac{t}{T} \\
& i_{2}(t)=i_{L} S q \frac{1}{T}\left(t+t_{\phi}\right),
\end{aligned}
$$

where $t_{\phi}$ is the time equivalent of the arbitrary phase difference between $i_{1}$ and $i_{2}$. Equation $(A-1-11)$ indicates that $S q(t)$ has a square wave form as shown in Fig. A-1-1. The capacitor voltage calculation is now written as

$$
v_{C}(t)=v_{C O}+\frac{i_{S}}{C} \int_{0}^{t} S q \frac{t}{T} d t+\frac{i_{L}}{C} \int_{0}^{t} S q \frac{1}{T}\left(t+t_{\phi}\right) d t .
$$

Using the quadrometric identities presented in Reference 9, Equation (A-1-13) simplifies to

$$
v_{C}(t)=-\frac{i_{S} T}{C} \operatorname{Tr} \frac{t}{T}-\frac{i_{L} T}{C} \operatorname{Tr} \frac{1}{T}\left(t+t_{\phi}\right)+\frac{i_{S} T}{4 C}+\frac{i_{L} T}{4 C}+v_{C 0} \cdot(A-1-14)
$$

A plot of $\operatorname{Tr}(t)$ function is shom in Fig. A-1-1. The instantaneous $i_{1}$ source output power is 

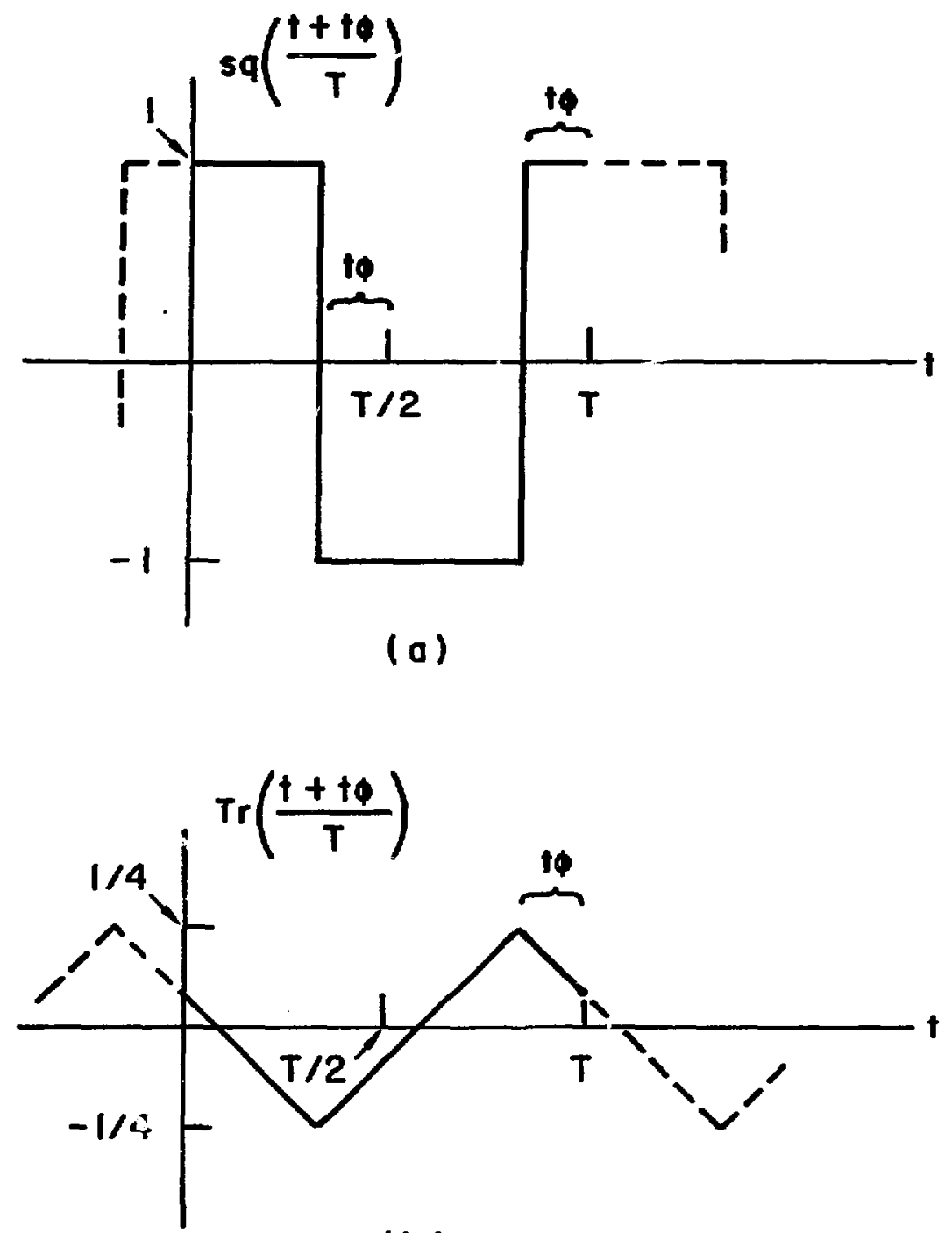

(b)

Fig. A-1-1. Plot of $S q(t)$ and $\operatorname{Tr}(t)$ at an Arbitrary Phase Shift. 


$$
\begin{aligned}
p_{1}(t)= & i_{1}(t) v_{C}(t) \\
= & -\frac{i_{S}^{2} T}{C} \operatorname{sq} \frac{t}{T} \operatorname{Tr} \frac{t}{T}-\frac{i_{S} i_{L}^{T}}{C} \operatorname{sq} \frac{t}{T} \operatorname{Tr} \frac{1}{T}\left(t+t_{\phi}\right) \\
& +i_{S}\left(\frac{i_{S} T}{4 C}+\frac{i_{L} T}{4 C}+v_{C O}\right) S q \frac{t}{T}
\end{aligned}
$$

The average power is

$$
\begin{aligned}
\left\langle p_{1}\right\rangle= & -\frac{i_{S}^{2}}{C} \int_{0}^{T} S q \frac{t}{T} \operatorname{Tr} \frac{t}{T} d t \\
& -\frac{i_{S}{ }^{i} L}{C} \int_{0}^{T} \operatorname{sq} \frac{t}{T} \operatorname{Tr} \frac{1}{T}\left(t+t_{\phi}\right) d t \\
& +\left(\frac{i_{S}^{2}}{4 C}+\frac{i_{S}{ }^{i} L}{4 C}+i_{S} v_{C o}\right) \int_{0}^{T} S q \frac{t}{T} d t
\end{aligned}
$$

Orthogonality of $\mathrm{Sq}$ and $\mathrm{Tr}$ functions, proven in Reference 9, shows that the first term of Equation $(A-1-16)$ is zero. The third term of this equation is the average of the $\mathrm{Sq}$ function which is easily seen to be zero. The second term integral is solved using the identities of Reference 9. After simplification the result is

$$
\left\langle p_{1}\right\rangle=\frac{1 S^{i} L}{c}\left(t_{\phi}-2 \frac{t_{\phi}^{2}}{T}\right) .
$$

Replacing the time difference, $t_{\phi}$, with phase angle difference, $\phi$, according to $t_{\phi} / T=\phi / 2 \pi$, will result in

$$
\left\langle p_{1}\right\rangle=\frac{{ }^{i} s^{i} L}{C}\left(\phi-\frac{\phi^{2}}{\pi}\right) .
$$

Equation $(A-1-18)$ is the same power as that shown in Equation (2-3). 


\section{APPENDIX 2 \\ Calculation of the First Transient Step in Frequency Shifting}

It is observed from the compone:t capacitor voltage in Fig. 12 that in frequency shiftiig, it is possible to get from the peak voltage at the old frequency, $A$, to the peak voltage at the new frequency, $B$, It: one step. This step, $\Delta t_{i}^{(2)}$ in Equation (2-35) can be intuitively derived by close examination of Fig. 12. The following is a simple calculation of this glantity in terms of the old and new switching intervals, $\Delta t_{1}$ and $\Delta t_{2} \cdot$ The vcltage at point $A$ is

$$
v_{C_{1 A}}=-\frac{i_{s} \Delta t_{1}}{2 C}
$$

The equation of the line during the transient step $\Delta t_{1}^{(2)}$ is

$$
v_{C 1}(t)=\frac{i_{S}}{C} t-\frac{i_{S} \Delta t_{1}}{2 C}
$$

where the time origin is assumed at point A. If point $B$ is the peak voltage at the new frequency, then its voltage should be

$$
v_{C 1 B}=\frac{i_{s} s_{2}}{2 C}
$$

The unknown time interval, $\Delta t_{1}^{(2)}$, on the line of Equation (A-2-2) shculd coincide with the voltage of Equation (A-2-3). Therefore, the unknown interval is calculated as follows:

$$
\begin{aligned}
& \frac{i_{S^{\Delta t}} t_{2}}{2 C}=\frac{i_{S}}{c} \Delta t_{1}^{(2)}-\frac{i_{S} \Delta t}{2 C} \\
& \Delta t_{1}^{(2)}=\frac{1}{2}\left(\Delta t_{1}+\Delta t_{2}\right) .
\end{aligned}
$$




\section{APPENDIX 3}

Operating Phase Threshold Calculation

The capacitor voltage, which determines the success or failure of a commutation event, is itself determined from two current components: the storage converter current, $i_{1}$, and the load converter current, $i_{2}$ (Fig. 2). A brief examination of the capacitor voltage, as in Fig. 16, will show that the SCR's of the converter whose inductor has the larger current will al.ways commutate successfully. Therefore, for the states $i_{L} / i_{S}<1$ only the commutations of the load SCRis, $S_{21}$ and $S_{24}$ or $S_{22}$ and $S_{23}$, need to be investigated. Furthermore, the commutaticn voltages for $\mathrm{s}_{21} \mathrm{~s}_{24}$ and $\mathrm{s}_{22} \mathrm{~s}_{23}$ are always symmetrical about the zero volt axis.* Therefore, investigation of only one of these voltages will reveal all the commutation outcomes. Investigating the commutation of $\mathrm{S}_{22} \mathrm{~S}_{23}$ means checking for the positiveness of the capacitor voltage at the commutation time. The capacitor voltage is

$$
v_{C}=v_{C l}+v_{C 2}
$$

Figure A-3-1 shows an arbitrary case of $v_{\mathrm{Cl}}$ and $v_{\mathrm{C} 2}$ when $i_{I} / i_{\mathrm{S}}<1$. The values of $v_{\mathrm{Cl}}$ and $\mathrm{v}_{\mathrm{C} 2}$ at the commutation instant, $t_{\mathrm{c}}$, can be found from the equations of their first line segments in Fig. A-3-1:

$$
\begin{aligned}
v_{C 1}(t) & =\frac{i_{S}}{C}\left(t-\frac{T}{4}\right) \\
v_{C L}(t) & =\frac{i_{L}}{C}\left(t-\frac{T}{4}+t_{\Phi}\right) \\
v_{C l}\left(t_{C}\right) & =v_{C l}\left(\frac{T}{2}-t_{\phi}\right)=\frac{i_{S}}{C}\left(\frac{T}{2}-t_{\phi}-\frac{T}{4}\right) \\
& =\frac{i_{S}}{C}\left(\frac{T}{4}-t_{\phi}\right)
\end{aligned}
$$

*SInce $v_{C}$ is symmetrical about the axis and $s_{21} s_{24}$ and $s_{22} s_{23}$ are $180^{\circ}$ apart from each other, their voltages must be symmetrical (see Fig. 16). 


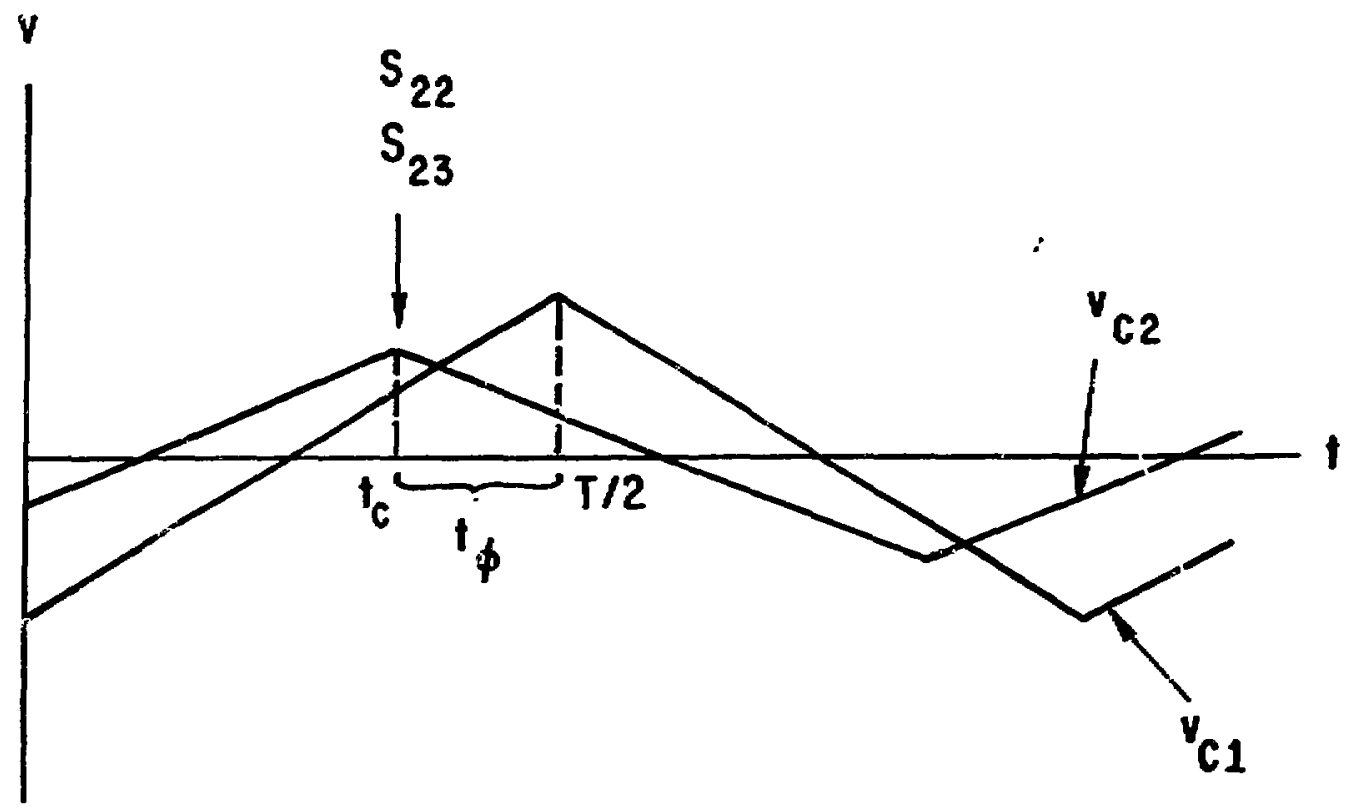

Fig. A-3-1. Capacitor component voltages in the vicinity of $\mathrm{S}_{22} \mathrm{~S}_{23}$ coumutation instant. 


$$
v_{C 2}\left(t_{C}\right)=v_{C 2}\left(\frac{T}{2}-t_{\phi}\right)=\frac{i_{L}}{C}\left(\frac{T}{2}-t_{\phi}-\frac{T}{4}+t_{\phi}\right)=\frac{i_{L} T}{4 C}
$$

The capacitor voltage at the commutation instant is

$$
v_{c}\left(t_{C}\right)=v_{C 1}\left(t_{c}\right)+v_{c 2}\left(t_{c}\right)=\frac{i_{S}}{C}\left(\frac{T}{4}-t_{\phi}\right)+\frac{i_{L} T}{4 C}
$$

For successful $\mathrm{S}_{22} \mathrm{~S}_{23}$ commutation we should have

$$
v_{C}>0+\frac{i_{S}}{C}\left(\frac{T}{4}-t_{\phi}\right)+\frac{i_{L} T}{4 C}>0 \rightarrow \frac{i_{L}}{i_{S}}>\left(\frac{4 t_{\phi}}{T}-1\right)
$$

After converting $t_{\phi}$ to $\phi$ according to $t_{\phi} / T=\phi / 360^{\circ}$ and simplifying the result, we will find

$$
\frac{\phi}{90}<\frac{i_{l}}{i_{S}}+1 \text {, }
$$

which is Equation (2-45) in the report.

A similar calculation can be done for the negative operating phases, $\phi<0^{\circ}$ at the states $i_{L} / i_{S}<1$. In this case, it is more convenient to investigate the commutation of $\mathrm{s}_{21} \mathrm{~s}_{24}$ in Fig. A-3-2. The task is to check tine negativeness of the capacitor voltage at point $t_{G}$ in the figure. The algebraic problem is identical to the one solved above, except the signs are reversei. The solution for the threshold condition will be

$$
\frac{\phi}{90}>-\left(\frac{i_{L}}{i_{S}}+1\right)
$$

which is shown as Equation (2-46) in the report. 


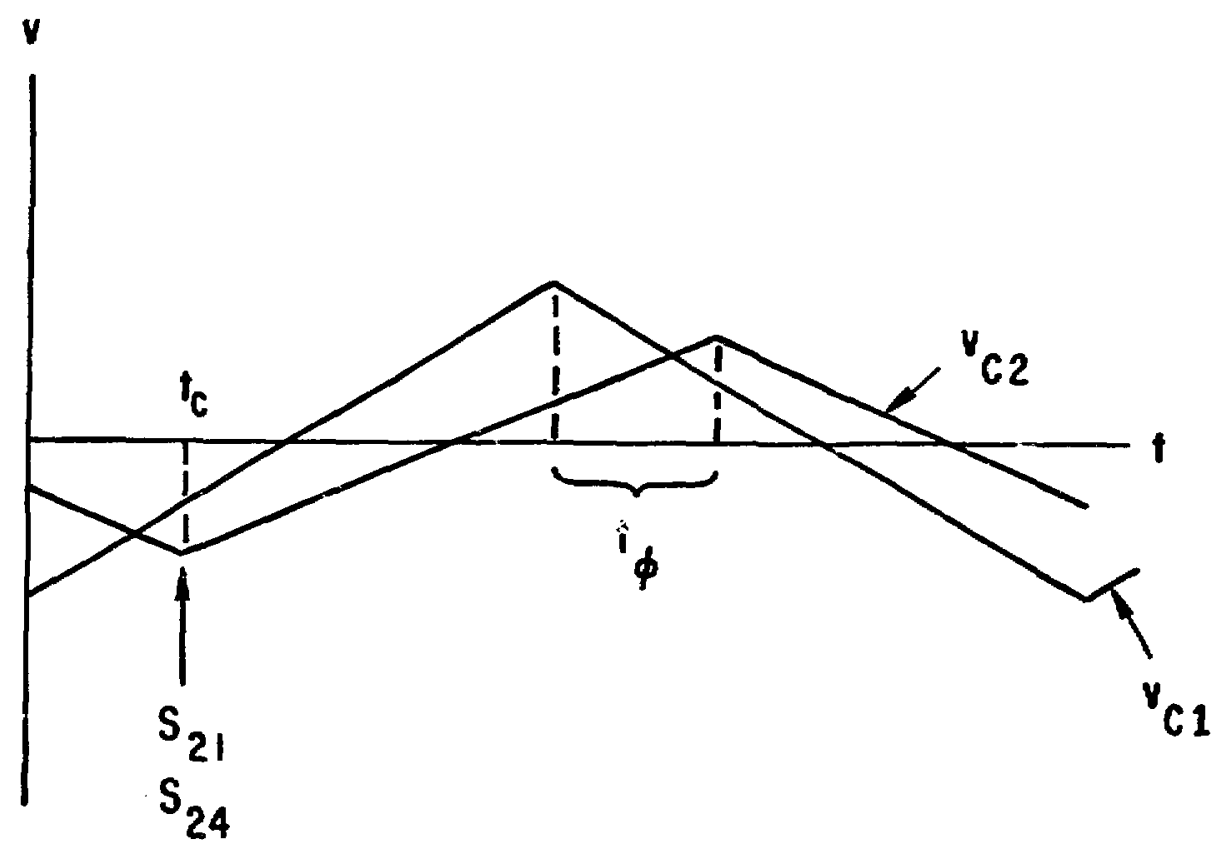

Fig. A-3-2. Capacitor componert vcltages in the vicinity of $\mathrm{S}_{21} \mathrm{~S}_{24}$ commutation instant. 
Distribution for ANL/FPP/TM-182

Internal:

C. Baker

C. Boley

J. Brooks

F. Cafasso

R. Clemmer

D. Ehst

K. Evans

P. Finn

Y. Gohar

L. Greenwood
D. Gruen

A. Hassanein

C. Johnson

J. Jung

S. Kim

R. Kustom (5)

R. Lari

B. Loomis

S. Majumdar

R. Mattas

B. Misra
R. Nygren

W. Praeg

J. Roberts

D. Smith

L. Turner

FP Program (15)

ANL Patent Dept.

ANL Contract File

ANL Libraries (2)

TIS Files (5)

\section{Externa1:}

DOE-TIC, for distribution per UC-20 (106)

Manager, Chicago Operations office, DOE

Special Committee for the Fusion Power Program:

S. Baron, Burns and Roe, Inc., Orade11, N. J.

H. K. Forsen, Bechtel Group, Inc., San Francisco

J. A. Maniscalco, TRH, Inc., Redondo Beach

G. H. Miley, U; IIlinois, Urbana

P. J. Reardon, Brookhaven National Lab.

P. H. Rutherford, Princeton U.

D. Steiner, Rensselaer Polytechnic Inst.

K. R. Symon, U. Wisconsin, Madison

K. I. Thomassen, Lavrence Livermore National Lab.

M. Ehsani, Texas A\&M U. (12) 